\title{
Application of analytic functions to the global solvabilty of the Cauchy problem for equations of Navier-Stokes
}

\section{Asset Durmagambetov}

Ministry of Education and Science of the Republic of Kazakhstan, Buketov Karaganda State University, Institute of Applied Mathematics, Buketov Karaganda, Kazakhstan; aset.durmagambetov@gmail.com

Received 14 December 2009; revised 29 January 2010; accepted 3 February 2010.

\begin{abstract}
The interrelation between analytic functions and real-valued functions is formulated in the work. It is shown such an interrelation realizes nonlinear representations for real-valued functions that allow to develop new methods of estimation for them. These methods of estimation are approved by solving the Cauchy problem for equations of viscous incompressible liquid.
\end{abstract}

Keywords: Shrödinger; Cauchy Problem; Navier-Stokes'; Inverse; Analytic Functions; Scattering Theory

\section{INTRODUCTION}

The work of L. Fadeyev dedicated to the many- dimensional inverse problem of scattering theory inspired the author of this article to conduct this research. The first results obtained by the author are described in the works [1-3]. This problem includes a number of subproblems which appear to be very interesting and complicated. These subproblems are thoroughly considered in the works of the following scientists: R. Newton [4], R. Faddeyev [5], R. Novikov and G. Khenkin [6], A. Ramm [3] and others. The latest advances in the theory of SIPM (Scattering Inverse Problem Method) were a great stimulus for the author as well as other researchers. Another important stimulus was the work of M. Lavrentyev on the application of analytic functions to Hydrodynamics. Only one-dimensional equations were integrated by SIPM. The application of analytic functions to Hydrodynamics is restricted only by bidimensional problems. The further progress in applying SIPM to the solution of nonlinear equations in R3 was hampered by the poor development of the three-dimensional inverse problem of scattering in comparison with the progress achieved in the work on the one-dimensional inverse problem of scattering and also by the difficulties the researchers encountered building up the corresponding Lax' pairs. It is easy to come to a conclusion that all the success in developing the theory of SIPM is connected with analytic functions, i.e., solutions to Schrodinger's equation. Therefore we consider Schrodinger's equation as an interrelation between real-valued functions and analytic functions, where real-valued functions are potentials in Schrodinger's equation and analytic functions are the corresponding eigenfunctions of the continuous spectrum of Schrodinger's operator. The basic aim of the paper is to study this interrelation and its application for obtaining new estimates to the solutions of the problem for Navier-Stokes' equations. We concentrated on formulating the conditions of momentum and energy conservation laws in terms of potential instead of formulating them in terms of wave functions. As a result of our study, we obtained non-trivial nonlinear relationships of potential. The effectiveness and novelty of the obtained results are displayed when solving the notoriously difficult Chauchy problem for Navier-Stokes' equations of viscous incompressible fluid.

\section{BASIC NOTIONS AND SUBSIDIARY STATEMENT}

Let us consider Shrödingerse equation

$$
-\Delta_{x} \varphi+q \varphi=|k|^{2} \varphi
$$

where $q$ is a bounded fast-decreasing function,

$$
k \in R^{3}, \quad|k|^{2}=\sum_{j=1}^{3}{ } k_{j}^{2} .
$$

Definition 1. Rolnik's Class $\boldsymbol{R}$ is a set of measurable functions $q$,

$$
\|q\|_{\mathbf{R}}=\int_{R^{6}} \nmid \frac{q(x) q(y)}{|x-y|^{2}} d x d y<\infty .
$$

It is considered to be a general definition ([7]).

Theorem 1. Suppose that $q \in \boldsymbol{R}$; then a exists a unique solution of Eq.1, with asymptotic form (2) as $|x| \rightarrow \infty$. 


$$
\begin{gathered}
\varphi_{ \pm}(k, x)=e^{i(k, x)}+ \\
+\frac{e^{ \pm i|k||x|}}{|x|} A_{ \pm}\left(k, k^{\prime}\right)+0\left(\frac{1}{|x|}\right)
\end{gathered}
$$

where

$$
\begin{gathered}
x \in R^{3}, k^{\prime}=|k| \frac{x}{|x|},(k, x)=\sum_{j=1}^{3} \nmid k_{j} x_{j}, \\
A_{ \pm}(k, \lambda)=\frac{1}{(2 \pi)^{3}} \int_{R^{3}} \uparrow q(x) \varphi_{ \pm}(k, x) e^{-i(\lambda, x)} d x .
\end{gathered}
$$

The proof of this theorem is in [7].

Consider the operators $H=-\Delta_{x}+q(x), H_{0}=-\Delta_{x}$ defined in the dense set $W_{2}^{2}\left(R^{3}\right)$ in the space $L_{2}\left(R^{3}\right)$. The operator $H$ is called Schrodinger's operator. Povzner [8] proved that the functions $\varphi \pm(k, x)$ form a complete orthonormal system of eigenfunctions of the continuous spectrum of the operator $H$, and the operator fills up the whole positive semi-axis. Besides the continuous spectrum the operator $H$ can have a finite number $N$ of negative eigenvalues Denote these eigenvalues by $-E_{j}^{2}$ and conforming normalized egenfunctions by

where $\psi_{j}\left(x,-E_{j}^{2}\right) \in L_{2}\left(R^{3}\right)$.

$$
\psi_{j}\left(x,-E_{j}^{2}\right)(j=\overline{1, N}),
$$

Theorem 2 (About Completeness). For any vector-function $f \in L_{2}\left(R^{3}\right)$ and eigenfunctions of the operator $H$, we have Parseval's identity

$$
|f|_{L_{2}}^{2}=\sum_{j=1}^{N} \uparrow\left|f_{j}\right|^{2}+\int_{R^{3}}{ }^{\uparrow}|\bar{f}(s)|^{2} d s
$$

where $f_{j}$ and $\bar{f}$ are Fourier coefficients in case of discrete of and continuous spectrum respectively.

The proof of this theorem is in [8].

Theorem 3 (Birman - Schwinger's Estimate). Suppose $q \in R$. Then the number of discrete eigenvalues of Shrödinger operator satisfies the estimate

$$
N(q) \leq \frac{1}{(4 \pi)^{2}} \int_{R^{3}} \uparrow \int_{R^{3}} \uparrow \frac{q(x) q(y)}{|x-y|^{2}} d x d y .
$$

The proof of this theorem is in [9].

Definition 2. [7]

$$
T_{ \pm}\left(k, k^{\prime}\right)=\frac{1}{(2 \pi)^{3}} \int_{R^{3}} \uparrow \varphi_{ \pm}\left(x, k^{\prime}\right) e^{\mp i(k, x)} q(x) d x .
$$

$T_{ \pm}(.,$.$) is called T-matrix. Let us take into consider-$ ation a series for $T_{ \pm}$:

$$
T_{ \pm}\left(k, k^{\prime}\right)=\sum_{n=0}^{\infty} \nmid T_{n_{ \pm}}\left(k, k^{\prime}\right),
$$

where

$$
T_{0_{ \pm}}\left(k, k^{\prime}\right)=\frac{1}{(2 \pi)^{3}} \int_{R^{3}} \nmid e^{i\left(k^{\prime} \mp k, x\right)} q(x) d x,
$$

$$
\begin{aligned}
& T_{n_{ \pm}}\left(k, k^{\prime}\right)=\frac{1}{(2 \pi)^{3}} \frac{(-1)^{n}}{(4 \pi)^{n}} \int_{R^{3(n+1)}} \uparrow e^{\mp i\left(k, x_{0}\right)} \\
& \times q\left(x_{0}\right) \frac{e^{ \pm i\left|k^{\prime}\right|\left|x_{0}-x_{1}\right|}}{\left|x_{0}-x_{1}\right|} q\left(x_{1}\right) \ldots q\left(x_{n-1}\right) \\
& \times \frac{e^{ \pm i\left|k^{\prime}\right|\left|x_{n-1}-x_{n}\right|}}{\left|x_{n-1}-x_{n}\right|} q\left(x_{n}\right) e^{i\left(k^{\prime}, x_{n}\right)} d x_{0} \ldots d x_{n} .
\end{aligned}
$$

As well as in [7] we formulate.

Definition 3. Series (4) is called Born's series.

Theorem 4. Let $q \in L_{1}\left(R^{3}\right) \cap \boldsymbol{R}$. If $P q P_{\boldsymbol{R}}^{2} \leq 4 \pi$, then Born's series for $T\left(k, k^{\prime}\right)$ converges as $k, k^{\prime} \in R^{3}$.

The proof of the theorem is in [7].

Definition 4. Suppose $q \in R$; then the function $A(k, \lambda)$, denoted by the following equality

$$
\begin{gathered}
A(k, l)=\frac{1}{(2 \pi)^{3}} \int_{R^{3}} \uparrow q(x) \\
\varphi_{+}(k, x) e^{-i(\lambda, x)} d x,
\end{gathered}
$$

is called scattering amplitude

Corollary 1. Scattering amplitude $A(k, \lambda)$ is equal to T-matrix

$$
\begin{gathered}
A(k, l)=T_{+}(l, k) \\
=\frac{1}{(2 \pi)^{3}} \int_{R^{3}} \uparrow q(x) \varphi_{+}(k, x) e^{-i(\lambda, x)} d x .
\end{gathered}
$$

The proof follows from definition 4 .

It is a well-known fact [5] that the solutions $\varphi+(k, x)$ and $\varphi-(k, x)$ of Eq.1 are linearly dependent

$$
\varphi+=S \varphi-
$$

where $S$ is a scattering operator with the nucleus $S(k, \lambda)$ of the form

$$
S(k, \lambda)=\int_{R^{3}} \uparrow \varphi_{+}(k, x) \varphi_{+}^{*}(\lambda, x) d x .
$$

Theorem 5. (Conservation Law of Impulse and Energy). Assume that $q \in \boldsymbol{R}$, then

$$
S S^{*}=I, S^{*} S=I \text {, }
$$

where I is anunit operator.

The proof is in [5].

Let us use the following definitions

$$
\begin{gathered}
\tilde{q}(k)=\int_{R^{3}} \uparrow q(x) e^{i(k, x)} d x, \\
\tilde{q}(k-\lambda)=\int_{R^{3}} \uparrow q(x) e^{i(k-\lambda, x)} d x, \\
\tilde{q}_{\mathrm{mv}}(k)=\int_{R^{3}}\lceil\tilde{q}(k-\lambda)) \delta\left(|k|^{2}-|\lambda|^{2}\right) d \lambda,
\end{gathered}
$$




$$
\begin{gathered}
\left.A_{\mathrm{mv}}(k)=\int_{R^{3}} \uparrow A(k, l)\right) \delta\left(|k|^{2}-|l|^{2}\right) d l, \\
\int_{\uparrow} f(k, l) d e_{k}=\int_{R^{3}} \uparrow f(k, l) \delta\left(|k|^{2}-|l|^{2}\right) d k, \\
\int \uparrow f(k, l) d e_{\lambda}=\int_{R^{3}} \uparrow f(k, l) \delta\left(k^{2}-|l|^{2}\right) d l,
\end{gathered}
$$

where $k, \lambda \in R^{3}$ and $e_{k}=\frac{k}{|k|}, e_{\lambda}=\frac{\lambda}{|\lambda|}$.

\section{ESTIMATE OF AMPLITUDE MAXIMUM}

Let us consider the problem of estimating the maximum of amplitude, i.e., $\max _{k \in R^{3}}|A(k, k)|$. Let us estimate the $n$ term of Born's series $\left|T_{n}(k, k)\right|$.

Lemma 1. $\left|T_{n}(k, k)\right|$ satisfies the inequality

$$
\begin{gathered}
\left|T_{n+1}(k, k)\right| \leq \frac{1}{(2 \pi)^{3}} \frac{1}{(4 \pi)^{n+1}} \\
\times \frac{\gamma^{n}}{(2 \pi)^{2(n+1)}} \int_{R^{3}} \times \frac{|\tilde{q}(k)|^{2}}{|k|^{2}} d k, \\
\gamma=C \delta\|q\|+4 \pi M \tilde{q} \delta, C \delta=2 \frac{\sqrt{\pi}}{\sqrt{\delta}}
\end{gathered}
$$

where $\delta$-is a small value, $C$ is a positive number, $M \tilde{q}=\max _{k \in R^{3}}|\tilde{q}|$.

Theorem 6. Suppose that $\gamma<16 \pi^{3}$, then $\max _{k \in R^{3}}|A(k, k)|$ satisfies the following estimate

$$
\max _{k \in R^{3}}|A(k, k)| \leq \frac{1}{(2 \pi)^{3}} \frac{1}{16 \pi^{3}-\gamma} \int_{R^{3}} \nmid \frac{|\tilde{q}(k)|^{2}}{|k|^{2}} d k,
$$

where $\gamma=C \delta\|q\|+4 \pi M \tilde{q} \delta, \delta$ is a small value,

$$
C \delta=2 \sqrt{\frac{\pi}{\delta}}, M \delta=\max _{k \in R^{3}}|\tilde{q}| \text {. }
$$

\section{REPRESENTATION OF FUNCTIONS BY ITS SPHERICAL AVERAGES}

Let us consider the problem of defining a function by its spherical average. This problem emerged in the course of our calculation and we shall consider it hereinafter.

Let us consider the following integral equation

$$
\int_{R^{3}}\left\lceil\tilde{q}(t) \delta\left(|t-k|^{2}-|k|^{2}\right) d t=f(2 k),\right.
$$

where $k, t \in R^{3}, \delta$ is Dirac's delta function,

$$
\left.\left.f \in W_{2}^{2}\left(R^{3}\right),|k|^{2}=\sum_{i=1}^{3}\right\rceil k_{i}^{2},(k, t)=\sum_{i=1}^{3}\right\rceil k_{i} t_{i} .
$$

Let us formulate the basic result.

Theorem 7. Suppose that $f \in W_{2}^{2}\left(R^{3}\right)$, then

$$
(2 \pi)^{2} \tilde{q}(r, \xi, \eta)
$$

$$
\begin{gathered}
=-\frac{1}{r} \frac{\partial^{2}}{\partial r^{2}} \int_{0}^{\pi} \uparrow \int_{0}^{2 \pi} \uparrow\left(f\left(\frac{2 r}{\left(e_{k}, e_{s}\right)}, e_{k}\right)\right. \\
\left.+f\left(\frac{2 r}{\left(e_{k}, e_{s}\right)},-e_{k}\right)\right) \frac{r^{2}}{\left(e_{k}, e_{s}\right)^{2}} \sin \theta d \theta d \varphi,
\end{gathered}
$$

where

$$
\begin{gathered}
f\left(\frac{2 r}{\left(e_{k}, e_{s}\right)}, e_{k}\right)=\tilde{q}\left(\frac{2 r}{\left(e_{k}, e_{s}\right)}, e_{k}\right), \\
\sin \theta d \theta d \varphi=d e_{k}, \\
\sin \xi d \xi d \eta=d e_{s}, \quad r=|t| .
\end{gathered}
$$

Theorem 8. Fourier transformation of the function $q$ satisfies the following estimate

$$
|\tilde{q}|_{L_{1}} \leq \frac{1}{4}\left|z \frac{\partial \tilde{q}_{m v}}{\partial z^{2}}\right|_{L_{1}}+2\left|\frac{\partial \tilde{q}_{m v}}{\partial z^{2}}\right|_{L_{1}}+\left|\frac{\tilde{q}_{m v}}{z}\right|_{L_{1}}{ }^{\prime}
$$

\section{CORRELATION OF AMPLITUDE AND WAVE FUNCTIONS}

We take the relationship for $\varphi_{+}, \varphi_{-}$from (3)

$$
\begin{aligned}
& \varphi_{+}(k, x)=\varphi_{-}(k, x) \\
& -2 \pi i \int_{R^{3}}{ }^{\top} \delta\left(|k|^{2}-|l|^{2}\right) \\
& \times A(k, \lambda) \varphi_{-}(\lambda, x) d \lambda .
\end{aligned}
$$

Let us denote new functions and operators we will use further

$$
\begin{gathered}
\varphi_{0}\left(\sqrt{z} e_{k}, x\right)=e^{i\left(\sqrt{z} e_{k}, x\right)}, \\
\Phi_{0}\left(\sqrt{z} e_{k}, x\right)=\varphi_{0}\left(\sqrt{z} e_{k}, x\right)+\varphi_{0}\left(-\sqrt{z} e_{k}, x\right), \\
\Phi_{+}\left(\sqrt{z} e_{k}, x\right)=\varphi_{+}\left(\sqrt{z} e_{k}, x\right)-e^{i\left(\sqrt{z} e_{k}, x\right)} \\
+\varphi_{+}\left(-\sqrt{z} e_{k}, x\right)-e^{-i\left(\sqrt{z} e_{k}, x\right),} \\
\Phi_{-}\left(\sqrt{z} e_{k}, x\right)=\varphi_{-}\left(\sqrt{z} e_{k}, x\right)-e^{i\left(\sqrt{z} e_{k}, x\right)} \\
+\varphi_{-}\left(-\sqrt{z} e_{k}, x\right)-e^{-i\left(\sqrt{z} e_{k}, x\right),} \\
D_{1} f=-2 \pi i \int_{R^{3}} \uparrow A(k, \lambda) \delta(z-l) f(\lambda, x) d \lambda, \\
D_{2} f=-2 \pi i \int_{R^{3}} \uparrow A(-k, \lambda) \delta(z-l) f(\lambda, x) d \lambda, \\
D_{3} f=D_{1} f+D_{2} f,
\end{gathered}
$$

where $z=|k|^{2}, l=|\lambda|^{2}, \pm k= \pm \sqrt{z} e_{k}$. Let us introduce the operators $T_{ \pm}, T$ for the function $f \in W_{2}^{1}(R)$ by the formulas

$$
T_{+} f=\frac{1}{\pi i} \lim _{\operatorname{Im} z \rightarrow 0} \int_{-\infty}^{\infty} \gamma \frac{f(\sqrt{s})}{s-z} d s,
$$

where $\operatorname{Im} z>0$,

where $\operatorname{Im} z<0$, 


$$
T f=\frac{1}{2}\left(T_{+}+T_{-}\right) f
$$

Use (4) and the symbols $e_{r}=\frac{k}{|k|}$ to come to Riemann' problem of finding a function $\Phi_{+}$, which is analytic by the variable $z$ in the top half plane, and the function $\Phi_{-}$, which is analytical on the variable $z$ in the bottom half plane by the specified jump of discontinuity $f$ onto the positive semi axis.

For the jump the discontinuity of an analytical function, we have the following equations

$$
\begin{gathered}
f=\Phi_{+}-\Phi_{-} \\
f=D_{3}\left[\Phi_{-}\right]-D_{3}\left[\varphi_{-}\right]
\end{gathered}
$$

where $\varphi_{-}=\varphi_{-}(-\lambda, x)$.

Theorem 9. Suppose that $q \in \boldsymbol{R}$,

$$
\left.\varphi_{ \pm}\right|_{x=0, z=0}=0 \text {; }
$$

then the functions

$$
\begin{gathered}
\Psi_{1}=\left.\Phi_{ \pm}\left(\sqrt{z} e_{k}, x\right)\right|_{x=0}-\left.\Phi_{0}\left(\sqrt{z} e_{k}, x\right)\right|_{x=0}, \\
\Psi_{2}=\left.T_{ \pm} f\right|_{x=0}
\end{gathered}
$$

are coincided according to the class of analytical functions, coincide with bounded derivatives all over the complex plane with a slit along the positive semi axis.

Lemma 2. There exists $0<|\varepsilon|<\infty$ such that it satisfies the following condition $\left.\varphi_{+}\right|_{x=0, z=0}=0$ holds for the potential of the form $v=\varepsilon q$, where $q \in \boldsymbol{R}$.

Now, we can formulate Riemann's problem. Find the analytic function $\Phi_{ \pm}$that satisfies (5), (6) and its solution is set by the following theorem.

Theorem 10. Assume that $q \in \boldsymbol{R}$,

$$
\left.\varphi_{ \pm}\right|_{x=0, z=0}=0 \text {, }
$$

then

$$
\begin{gathered}
\Phi_{ \pm}=T_{ \pm} f+\Phi_{0}, \\
f=D_{3}\left[f\left[T_{-} f+\Phi_{0}\right]\right]-D_{3} \varphi_{-},
\end{gathered}
$$

where $\varphi_{-}=\varphi_{-}(-\lambda, x)$.

Lemma 3. Suppose that $q \in \boldsymbol{R},\left.\varphi_{ \pm}\right|_{x=0, z=0}=0$; then

$$
\left.\Delta_{x} T_{ \pm}[f]\right|_{x=0}=\left.T_{ \pm} \Delta_{x}[f]\right|_{x=0} .
$$

Theorem 11. Suppose that $q \in \boldsymbol{R}$,

$$
\left.\varphi_{ \pm}\right|_{x=0, z=0}=0, q(0) \neq 0 \text {, }
$$

then

$$
\begin{gathered}
\left.q(0) f\right|_{x=0}=D_{3} T_{-}\left[\left.q f\right|_{x=0}\right. \\
-\left.D_{3}\left[q \varphi_{-}\right]\right|_{x=0}+\left.D_{3} \int_{0} \uparrow f d s\right|_{x=0} .
\end{gathered}
$$

\section{AUXILIARY PROPOSITIONS}

For wave functions let us use integral representations following from Lippman-Schwinger's theorem

$$
\begin{gathered}
\varphi_{ \pm}(k, x)=e^{i(k, x)} \\
+\frac{1}{4 \pi} \int_{R^{3}} \nmid \frac{e^{ \pm i \sqrt{z}|x-y|}}{|x-y|} q(y) \varphi_{ \pm}(k, y) d y, \\
\varphi_{ \pm}(-k, x)=e^{-i(k, x)} \\
+\frac{1}{4 \pi} \int_{R^{3}} \nmid \frac{e^{\mp i \sqrt{z}|x-y|}}{|x-y|} q(y) \varphi_{ \pm}(-k, y) d y .
\end{gathered}
$$

Lemma 4. Suppose that $q \in \boldsymbol{R}$,

$$
\left.\varphi_{ \pm}\right|_{x=0, z=0}=0 \text {; }
$$

then

$$
\begin{gathered}
A\left(k, k^{\prime}\right)=c_{0} \tilde{q}\left(k-k^{\prime}\right) \\
+\frac{c_{0}}{4 \pi} \int_{R^{3}} \uparrow \int_{R^{3}} \uparrow e^{-i\left(k^{\prime}, x\right)} q(x) \frac{e^{i \sqrt{z}|x-y|}}{|x-y|} \\
\times q(y) e^{i(k, y)} d y d x+A_{3}\left(k, k^{\prime}\right), \\
A\left(-k, k^{\prime}\right)=c_{0} \tilde{q}\left(-k-k^{\prime}\right) \\
+\frac{c_{0}}{4 \pi} \int_{R^{3}} \uparrow \int_{R^{3}} \uparrow e^{-i\left(k^{\prime}, x\right)} q(x) \frac{e^{-i \sqrt{z}|x-y|}}{|x-y|} \\
\times q(y) e^{-i(k, y)} d y d x+A_{3}\left(-k, k^{\prime}\right),
\end{gathered}
$$

where $c_{0}=\frac{1}{(2 \pi)^{2}}$, and $A_{3}\left(k, k^{\prime}\right), A_{3}\left(-k, k^{\prime}\right)$ are terms of order higher than 2 with regards to $q$.

Theorem 12 (Parseval). The functions

$$
f, g \in L_{2}\left(R^{3}\right)
$$

satisfy the equation

$$
(f, g)=c_{0}\left(\tilde{f}, \tilde{g}^{*}\right),
$$

where $(\because$,$) is a scalar product and c_{0}=\frac{1}{(2 \pi)^{3}}$.

Lemma 5. Suppose that $q \in \boldsymbol{R},\left.\varphi_{ \pm}\right|_{x=0, z=0}=0$, then

$$
\begin{gathered}
A\left(k, k^{\prime}\right)=c_{0} \tilde{q}\left(k-k^{\prime}\right) \\
-c_{0}^{2} \int_{R^{3}} \uparrow \frac{\tilde{q}(k+p) \tilde{q}\left(p-k^{\prime}\right)}{|p|^{2}-z-i 0} d p \\
+A_{3}\left(k, k^{\prime}\right), \\
A\left(-k, k^{\prime}\right)=c_{0} \tilde{q}\left(-k-k^{\prime}\right) \\
-c_{0}^{2} \int_{R^{3}} \nmid \frac{\tilde{q}(-k+p) \tilde{q}\left(p-k^{\prime}\right)}{|p|^{2}-z-i 0} d p \\
+A_{3}\left(-k, k^{\prime}\right) .
\end{gathered}
$$

Corollary 2. Suppose that $q \in \boldsymbol{R}$,

$$
\left.\varphi_{ \pm}\right|_{x=0, z=0}=0 \text {, }
$$

then

$$
\begin{gathered}
A_{\mathrm{mv}}(k)=c_{0} \tilde{q}_{\mathrm{mv}}(k) \\
-c_{0}^{2} \frac{\sqrt{z}}{2} \int_{0}^{\pi} \uparrow \int_{0}^{2 * \pi} \uparrow \int_{R^{3}} \uparrow \frac{\tilde{q}(k+p) \tilde{q}\left(p-k^{\prime}\right)}{|p|^{2}-z-i 0} d p d e_{k^{\prime}}+A_{3 \mathrm{mv}}(k)
\end{gathered}
$$


where

$$
A_{3 \mathrm{mv}}(k)=\int_{R^{3}} \uparrow A_{3}\left(k, k^{\prime}\right) \delta\left(z-\left|k^{\prime}\right|^{2}\right) d k^{\prime}
$$

and

$$
\begin{gathered}
A_{\mathrm{mv}}(-k)=c_{0} \tilde{q}_{\mathrm{mv}}(-k) \\
-c_{0}^{2} \frac{\sqrt{z}}{2} \int_{0}^{\pi} \uparrow \int_{0}^{2 * \pi} \uparrow \int_{R^{3}} \uparrow \frac{\tilde{q}(-k+p) \tilde{q}\left(p-k^{\prime}\right)}{|p|^{2}-z-i 0} d p d e_{k^{\prime}} \\
+A_{3 \mathrm{mv}}(-k),
\end{gathered}
$$

where

$$
A_{3 \mathrm{mv}}(-k)=\int_{R^{3}} \nmid A_{3}\left(-k, k^{\prime}\right) \delta\left(z-\left|k^{\prime}\right|^{2}\right) d k^{\prime} .
$$

Lemma 6. Suppose that $q \in R$ and $x=0$, then

$$
\begin{gathered}
\varphi_{ \pm}(k, 0)=1+\frac{1}{4 \pi} \int_{R^{3}} \nmid \frac{e^{ \pm i \sqrt{z}|y|}}{|y|} q(y) e^{i(k, y)} d y \\
+\frac{1}{(4 \pi)^{2}} \int_{R^{3}} \uparrow \int_{R^{3}} \nmid \frac{e^{ \pm i \sqrt{z}|y|}}{|y|} q(y) \frac{e^{ \pm i \sqrt{z}|y-t|}}{|y-t|} \\
\times q(t) e^{i(k, t)} d t d y+\varphi_{ \pm}^{(3)}(k, 0),
\end{gathered}
$$

where $\varphi_{ \pm}^{(3)}(k, 0)$ are terms of order higher than 2 with regards to q., i.e.,

$$
\begin{gathered}
\varphi_{ \pm}^{(3)}(k, x)=\frac{1}{(4 \pi)^{3}} \int_{R^{3}} \uparrow \int_{R^{3}} \uparrow \int_{R^{3}} \nmid \frac{e^{ \pm i \sqrt{z}|x-y|}}{|x-y|} q(y) \\
\times \frac{e^{ \pm i \sqrt{z}|y-t|}}{|y-t|} q(t) \frac{e^{ \pm i \sqrt{z}|t-s|}}{|t-s|} q(s) \varphi_{ \pm}(k, s) d s d t d y .
\end{gathered}
$$

and

$$
\begin{gathered}
\varphi_{ \pm}(-k, 0)=1+\frac{1}{4 \pi} \int_{R^{3}} \nmid \frac{e^{\mp i \sqrt{z}|y|}}{|y|} q(y) e^{-i(k, y)} d y \\
+\frac{1}{(4 \pi)^{2}} \int_{R^{3}} \uparrow \int_{R^{3}} \uparrow \frac{e^{\mp i \sqrt{z}|y|}}{|y|} q(y) \frac{e^{\mp i \sqrt{z}|y-t|}}{|y-t|} q(t) \\
\times e^{-i(k, t)} d t d y+\varphi_{ \pm}^{(3)}(-k, 0),
\end{gathered}
$$

where $\varphi_{ \pm}^{(3)}(-k, 0)$ are terms of order higher than 2 with regards to q., i.e.,

$$
\begin{gathered}
\varphi_{ \pm}^{(3)}(-k, x)=\frac{1}{(4 \pi)^{3}} \int_{R^{3}} \uparrow \int_{R^{3}} \uparrow \int_{R^{3}} \nmid \frac{e^{\mp i \sqrt{z}|x-y|}}{|x-y|} q(y) \\
\times \frac{e^{\mp i \sqrt{z}|y-t|}}{|y-t|} q(t) \frac{e^{\mp i \sqrt{z}|t-s|}}{|t-s|} q(s) \varphi_{ \pm}(-k, s) d s d t d y .
\end{gathered}
$$

Lemma 7. Suppose that $q \in \boldsymbol{R},\left.\varphi_{ \pm}\right|_{x=0, z=0}=0$, then

$$
\varphi_{ \pm}(k, 0)=1-c_{0} \int_{R^{3}} \nmid \frac{\tilde{q}(k+p)}{|p|^{2}-z \mp i 0} d p
$$

$$
\begin{gathered}
+c_{0}^{2} \int_{R^{3}} \uparrow \frac{\tilde{q}(k+p)}{\left(|p|^{2}-z \bar{\mp} 0\right)} \\
\times \int_{R^{3}} \uparrow \frac{\tilde{q}\left(p+p_{1}\right)}{\left(\left|p_{1}\right|^{2}-z \mp i 0\right)} d p_{1} d p+\varphi_{ \pm}^{(3)}(k, 0) \\
\varphi_{ \pm}(-k, 0)=1-c_{0} \int_{R^{3}} \uparrow \frac{\tilde{q}(-k+p)}{|p|^{2}-z \mp i 0} d p \\
+c_{0}^{2} \int_{R^{3}} \uparrow \frac{\tilde{q}(-k+p)}{\left(|p|^{2}-z \bar{\mp} i 0\right)} \\
\times \int_{R^{3}} \uparrow \frac{\tilde{q}\left(p+p_{1}\right)}{\left(\left|p_{1}\right|^{2}-z \bar{\mp} i 0\right)} d p_{1} d p+\varphi_{ \pm}^{(3)}(-k, 0)
\end{gathered}
$$

Lemma 8. Suppose that $q \in R, x=0$; then

$$
\begin{aligned}
& F(k, 0)=-\pi i c_{0} \sqrt{z} \int_{0}^{\pi} \uparrow \int_{0}^{2 \pi} \uparrow \tilde{q}\left(k-\sqrt{z} e_{p}\right) d e_{p} \\
&+\pi i c_{0}^{2} \sqrt{z} \int_{0}^{\pi} \uparrow \int_{0}^{2 \pi} \uparrow V \cdot p \cdot \int_{R^{3}} \uparrow \frac{\tilde{q}\left(k-\sqrt{z} e_{p}\right)}{\left|p_{1}\right|^{2}-z} \\
& \times \tilde{q}\left(-\sqrt{z} e_{p}-p_{1}\right) d p_{1} d e_{p} \\
&+\pi i c_{0}^{2} \sqrt{z} V \cdot p \cdot \int_{R^{3}} \uparrow \int_{0}^{\pi} \uparrow \int_{0}^{2 \pi} \uparrow \frac{\tilde{q}(k-p)}{|p|^{2}-z} \\
& \quad \times \tilde{q}\left(-p-\sqrt{z} e_{p_{1}}\right) d e_{p_{1}} d p \\
&+\varphi_{+}^{(3)}(k, 0)-\varphi_{-}^{(3)}(k, 0) .
\end{aligned}
$$

and

$$
F(-k, 0)=-\pi i c_{0} \sqrt{z} \int_{0}^{\pi} \uparrow \int_{0}^{2 \pi} \uparrow \tilde{q}\left(-k-\sqrt{z} e_{p}\right) d e_{p}
$$

$$
\begin{aligned}
+\pi i c_{0}^{2} \sqrt{z} \int_{0}^{\pi} \uparrow \int_{0}^{2 \pi} \uparrow V \cdot p . \int_{R^{3}} \uparrow \frac{\tilde{q}\left(-k-\sqrt{z} e_{p}\right)}{\left|p_{1}\right|^{2}-z} \\
\times \tilde{q}\left(-\sqrt{z} e_{p}-p_{1}\right) d p_{1} d e_{p} \\
+\pi i c_{0}^{2} \sqrt{z} V \cdot p \cdot \int_{R^{3}} \uparrow \int_{0}^{\pi} \uparrow \int_{0}^{2 \pi} \uparrow \frac{\tilde{q}(-k-p)}{|p|^{2}-z} \\
\times \tilde{q}\left(-p-\sqrt{z} e_{p_{1}}\right) d e_{p_{1}} d p \\
+\varphi_{+}^{(3)}(-k, 0)-\varphi_{-}^{(3)}(-k, 0) .
\end{aligned}
$$

\section{TWO REPRESENTATIONS OF SCATTERING AMPLITUDE}

Lemma 9. Suppose that $f \in W_{2}^{1}(R)$, then

$$
T_{ \pm} f=\mp f+T f .
$$

Lemma 10. Suppose that $q \in \boldsymbol{R},\left.\varphi_{ \pm}\right|_{x=0, z=0}=0$, then

$$
f(k, 0)=F(k, 0)+F(-k, 0) .
$$


Lemma 11. Suppose that $q \in \boldsymbol{R},\left.\varphi_{ \pm}\right|_{x=0, z=0}=0$, then

$$
\begin{aligned}
& A_{\mathrm{mv}}(k)+A_{\mathrm{mv}}(-k)=c_{0}\left(\tilde{q}_{\mathrm{mv}}(k)+\tilde{q}_{\mathrm{mv}}(-k)\right) \\
& +\pi i c_{0}^{2} \sqrt{z} \int_{0}^{\pi} \uparrow \int_{0}^{2 \pi} \uparrow\left(\tilde{q}\left(k-\sqrt{z} e_{\lambda}\right)+\tilde{q}\left(-k-\sqrt{z} e_{\lambda}\right)\right) \\
& \times \tilde{q}_{\mathrm{mv}}\left(\sqrt{z} e_{\lambda}\right) d e_{\lambda} \\
& +\pi i c_{0}^{2} \frac{\sqrt{z}}{2} \int_{0}^{\pi} \uparrow \int_{0}^{2 \pi} \uparrow\left(\tilde{q}\left(k-\sqrt{z} e_{\lambda}\right)+\tilde{q}\left(-k-\sqrt{z} e_{\lambda}\right)\right) \\
& \times \tilde{q}_{\mathrm{mv}}\left(-\sqrt{z} e_{\lambda}\right) d e_{\lambda} \\
& -\pi i c_{0}^{2} \sqrt{z} \int_{0}^{\pi} \uparrow \int_{0}^{2 \pi} \uparrow\left(\tilde{q}\left(k-\sqrt{z} e_{\lambda}\right)+\tilde{q}\left(-k-\sqrt{z} e_{\lambda}\right)\right) \\
& \times\left(T\left[\tilde{q}_{\mathrm{mv}}\right]\left(\sqrt{z} e_{\lambda}\right)+T\left[\tilde{q}_{\mathrm{mv}}\right]\left(-\sqrt{z} e_{\lambda}\right)\right) d e_{\lambda} \\
& -c_{0}^{2} \sqrt{z} \int_{0}^{\pi} \uparrow \int_{0}^{2 \pi} \uparrow\left(\tilde{q}\left(k-\sqrt{z} e_{\lambda}\right)+\tilde{q}\left(-k-\sqrt{z} e_{\lambda}\right)\right) \\
& \times V \cdot p \cdot \int_{R^{3}} \nmid \frac{\tilde{q}\left(-\sqrt{z} e_{\lambda}-p\right)}{|p|^{2}-z} d p d e_{\lambda} \\
& +c_{0}^{2} \frac{\sqrt{z}}{2} V \cdot p . \int_{R^{3}} \uparrow \int_{0}^{\pi} \uparrow \int_{0}^{2 \pi} \uparrow \frac{\tilde{q}(k-\lambda)+\tilde{q}(-k-\lambda)}{l-z} \\
& \times \tilde{q}\left(-l-\sqrt{z} e_{p}\right) d e_{p} d \lambda-2 \pi i\left(F^{(3)}(k, 0)\right. \\
& \left.+F^{(3)}(-k, 0)+Q_{3}(k, 0)+Q^{(3)}(k, 0)\right),
\end{aligned}
$$

where $Q_{3}(k, 0), Q^{(3)}(k, 0)$ are defined by formulas

$$
\begin{gathered}
Q_{3}(k, 0)=-4 \pi^{2} c_{0}^{2} \int_{R^{3}} \uparrow\left(A_{2}(k, \lambda)+A_{2}(-k, \lambda)\right) \\
\times \delta(z-l)\left(\tilde{q}_{\mathrm{mv}}(\lambda)+\tilde{q}_{\mathrm{mv}}(-\lambda)\right) d \lambda \\
+2 \pi i c_{0} \int_{R^{3}} \uparrow\left(A_{2}(k, \lambda)+A_{2}(-k, \lambda)\right) \delta(z-l) \\
\times f_{2}(l, 0) d l+4 \pi^{2} c_{0}^{2} \int_{R^{3}} \uparrow\left(A_{2}(k, \lambda)+A_{2}(-k, \lambda)\right) \\
\times \delta(z-l)\left(T\left[\tilde{q}_{\mathrm{mv}}\right](\lambda)+T\left[\tilde{q}_{\mathrm{mv}}\right](-\lambda)\right) d \lambda \\
-2 \pi i c_{0} \int_{R^{3}} \uparrow\left(A_{2}(k, l)+A_{2}(-k, l)\right) \\
\times \delta(z-l) T\left[f_{2}\right](\lambda, 0) d \lambda . \\
Q^{(3)}(k, 0)=2 \pi i c_{0}^{2} \int_{R^{3}} \uparrow(\tilde{q}(k-\lambda)+\tilde{q}(-k-\lambda)) \\
\times \delta(z-l) \varphi_{-}^{(2)}(-\lambda, 0) d \lambda \\
+2 \pi i c_{0}^{2} \int_{R^{3}} \uparrow\left(A_{2}(k, \lambda)+A_{2}(-k, \lambda)\right)
\end{gathered}
$$

$$
\begin{gathered}
\times \delta(z-l)\left(\int_{R^{3}} \uparrow \frac{\tilde{q}(-\lambda-p)}{|p|^{2}-l+i 0} d p\right. \\
\left.+\varphi_{-}^{(2)}(-l, 0)\right) d \lambda .
\end{gathered}
$$

correspondingly,

$$
\begin{gathered}
F^{(3)}(k, 0)=\varphi_{+}^{(3)}(k, 0)-\varphi_{-}^{(3)}(k, 0), \\
F^{(3)}(-k, 0)=\varphi_{+}^{(3)}(-k, 0)-\varphi_{-}^{(3)}(-k, 0),
\end{gathered}
$$

and $\varphi_{ \pm}^{(3)}( \pm k, 0)$ are terms of order 3 and higher w.r.t. $\tilde{q}$ in the representations (7), (8).

Lemma 12. Suppose that $q \in \boldsymbol{R},\left.\varphi_{ \pm}\right|_{x=0, z=0}=0$, then

$$
\begin{gathered}
A_{\mathrm{mv}}(k)+A_{\mathrm{mv}}(-k) \\
=-\frac{i \sqrt{z}}{4 \pi q(0)} \int_{0}^{\pi} \uparrow \int_{0}^{2 \pi} \uparrow\left(A\left(k, \sqrt{z} e_{\lambda}\right)+A\left(-k, \sqrt{z} e_{\lambda}\right)\right) \\
\times \int_{0}^{\infty} \uparrow f\left(s e_{\lambda}, 0\right) d s d e_{\lambda} .
\end{gathered}
$$

\section{NONLINEAR REPRESENTATION OF POTENTIAL}

Let us proceed to the construction of potential nonlinear representation.

Lemma 13. Assume that $q \in \boldsymbol{R},\left.\varphi_{ \pm}\right|_{x=0, z=0}=0$; then

$$
\begin{gathered}
\tilde{q}_{\mathrm{mv}}(k)+\tilde{q}_{\mathrm{mv}}(-k) \\
=-\pi i c_{0} \sqrt{z} \int_{0}^{\pi} \uparrow \int_{0}^{2 \pi}\left(\tilde{q}\left(k-\sqrt{z} e_{\lambda}\right)\right. \\
\left.+\tilde{q}\left(-k-\sqrt{z} e_{\lambda}\right)\right) \tilde{q}_{\mathrm{mv}}\left(\sqrt{z} e_{\lambda}\right) d e_{\lambda} \\
-\pi i c_{0} \frac{\sqrt{z}}{2} \int_{0}^{\pi} \uparrow \int_{0}^{2 \pi} \uparrow\left(\tilde{q}\left(k-\sqrt{z} e_{\lambda}\right)+\tilde{q}\left(-k-\sqrt{z} e_{\lambda}\right)\right) \\
+\pi i c_{0} \sqrt{z} \int_{0}^{\pi} \int_{0}^{2 \pi} \uparrow\left(\tilde{q}\left(k-\sqrt{z} e_{\lambda}\right)+\tilde{q}\left(-k-\sqrt{z} e_{\lambda}\right)\right) \\
\times\left(T\left[\tilde{q}_{\mathrm{mv}}\right]\left(\sqrt{z} e_{\lambda}\right)+T\left[\tilde{q}_{\mathrm{mv}}\right]\left(-\sqrt{z} e_{\lambda}\right)\right) d e_{\lambda}\left(-\sqrt{z} e_{\lambda}\right) d e_{\lambda} \\
-c_{0} \sqrt{z} \int_{0}^{\pi} \int_{0}^{2 \pi} \uparrow\left(\tilde{q}\left(k-\sqrt{z} e_{\lambda}\right)+\tilde{q}\left(-k-\sqrt{z} e_{\lambda}\right)\right) \\
\times V \cdot p \cdot \int_{R^{3}} \uparrow \frac{\tilde{q}\left(-\sqrt{z} e_{\lambda}-p\right)}{|p|^{2}-z} d p d e_{\lambda} \\
-c_{0} \frac{\sqrt{z}}{2} V \cdot p \cdot \int_{R^{3}} \uparrow \int_{0}^{\pi} \uparrow \int_{0}^{2 \pi}(\tilde{q}(k-\lambda)+\tilde{q}(-k-\lambda)) \\
l-z
\end{gathered}
$$




$$
\begin{gathered}
\times \tilde{q}\left(-l-\sqrt{z} e_{p}\right) d e_{p} d \lambda \\
-\frac{i \sqrt{z}}{4 \pi c_{0} q(0)} \int_{0}^{\pi} \uparrow \int_{0}^{2 \pi} \uparrow\left(A\left(k, \sqrt{z} e_{\lambda}\right)+A\left(-k, \sqrt{z} e_{\lambda}\right)\right) \\
\times \int_{0}^{\infty} \uparrow f\left(s e_{\lambda}, 0\right) d s d e_{\lambda}+\frac{2 \pi i}{c_{0}}\left(F^{(3)}(k, 0)\right. \\
\left.+F^{(3)}(-k, 0)+Q_{3}(k, 0)+Q^{(3)}(k, 0)\right),
\end{gathered}
$$

where $Q_{3}(k, 0), Q^{(3)}(k, 0)$ are defined by Eqs.9 and 10 accordingly,

$$
\begin{gathered}
F^{(3)}(k, 0)=\varphi_{+}^{(3)}(k, 0)-\varphi_{-}^{(3)}(k, 0), \\
F^{(3)}(-k, 0)=\varphi_{+}^{(3)}(-k, 0)-\varphi_{-}^{(3)}(-k, 0),
\end{gathered}
$$

and $\varphi_{ \pm}^{(3)}( \pm k, 0)$ are term of order 3 and higher w.r.t. $\tilde{q}$ in representations (7), (8).

Lemma 14. Suppose that $q \in \boldsymbol{R},\left.\varphi_{ \pm}\right|_{x=0, z=0}=0$, then

$$
\begin{gathered}
\text { V.p. } \int_{R^{3}} \uparrow \int_{0}^{\pi} \uparrow \int_{0}^{2 \pi} \uparrow \frac{(\tilde{q}(k-\lambda)+\tilde{q}(-k-\lambda))}{l-z} \\
\times \tilde{q}\left(-l-\sqrt{z} e_{p}\right) d e_{p} d l \\
=\pi i \int_{0}^{\pi} \uparrow \int_{0}^{2 \pi} \uparrow\left(\tilde{q}\left(k-\sqrt{z} e_{\lambda}\right)+\tilde{q}\left(-k-\sqrt{z} e_{\lambda}\right)\right) \\
\times \tilde{q}_{\mathrm{mv}}\left(-\sqrt{z} e_{\lambda}\right) d e_{\lambda} .
\end{gathered}
$$

Lemma 15. Let $\tilde{q} \in W_{2}^{1}(R)$ and $q \in R$, then

$$
\begin{aligned}
& \int_{0}^{\pi} \uparrow \int_{0}^{2 \pi} \uparrow\left(\tilde{q}\left(k-\sqrt{z} e_{\lambda}\right)+\tilde{q}\left(-k-\sqrt{z} e_{\lambda}\right)\right) \\
& \times\left(T\left[\tilde{q}_{\mathrm{mv}}\right]\left(\sqrt{z} e_{\lambda}\right)+T\left[\tilde{q}_{\mathrm{mv}}\right]\left(-\sqrt{z} e_{\lambda}\right)\right) d e_{\lambda} \\
& =\int_{0}^{\pi} \uparrow \int_{0}^{2 \pi} \uparrow\left(\tilde{q}\left(k-\sqrt{z} e_{\lambda}\right)+\tilde{q}\left(-k-\sqrt{z} e_{\lambda}\right)\right) \\
& \times\left(\tilde{q}_{\mathrm{mv}}\left(\sqrt{z} e_{\lambda}\right)+\tilde{q}_{\mathrm{mv}}\left(-\sqrt{z} e_{\lambda}\right)\right) d e_{\lambda}, \\
& \int_{0}^{\pi} \int_{0}^{2 \pi} \uparrow\left(\tilde{q}\left(k-\sqrt{z} e_{\lambda}\right)+\tilde{q}\left(-k-\sqrt{z} e_{\lambda}\right)\right) \\
& \times V \cdot p . \int_{R^{3}} \uparrow \frac{\tilde{q}\left(-\sqrt{z} e_{\lambda}-p\right)}{|p|^{2}-z} d p d e_{\lambda} \\
& =\pi i \int_{0}^{\pi} \uparrow \int_{0}^{2 \pi} \uparrow\left(\tilde{q}\left(k-\sqrt{z} e_{\lambda}\right)+\tilde{q}\left(-k-\sqrt{z} e_{\lambda}\right)\right) \\
& \times \tilde{q}_{\mathrm{mv}}\left(-\sqrt{z} e_{\lambda}\right) d e_{\lambda} .
\end{aligned}
$$

Theorem 14. Let $q \in \boldsymbol{R},\left.\varphi_{ \pm}\right|_{x=0, z=0}=0$, then

$$
\tilde{q}_{\mathrm{mv}}(k)+\tilde{q}_{\mathrm{mv}}(-k)
$$

$$
\begin{aligned}
& =-\pi i c_{0} \sqrt{z} \int_{0}^{\pi} \uparrow \int_{0}^{2 \pi} \uparrow\left(\tilde{q}\left(k-\sqrt{z} e_{\lambda}\right)+\tilde{q}\left(-k-\sqrt{z} e_{\lambda}\right)\right) \\
& \times \tilde{q}_{\mathrm{mv}}\left(-\sqrt{z} e_{\lambda}\right) d e_{\lambda}+\mu(k), \\
& \mu(k)=\frac{2 \pi i}{c_{0}}\left(F^{(3)}(k, 0)+F^{(3)}(-k, 0)\right. \\
& \left.+Q_{3}(k, 0)+Q^{(3)}(k, 0)\right),
\end{aligned}
$$

where $c_{0}=4 \pi$.

Theorem 15. Suppose $q \in \boldsymbol{R},\left.\varphi_{ \pm}\right|_{x=0, z=0}=0$; then

$$
\begin{gathered}
\mu(k)=\sqrt{z} \int_{0}^{\pi} \uparrow \int_{0}^{2 \pi} \uparrow \int_{0}^{\pi} \uparrow \int_{0}^{2 \pi} \uparrow\left(\tilde{q}\left(-k-\sqrt{z} e_{\lambda}\right)\right. \\
\left.+\tilde{q}\left(k-\sqrt{z} e_{\lambda}\right)\right) \tilde{q}\left(\sqrt{z} e_{\lambda}-\sqrt{z} e_{S}\right) \\
\times \mu_{0}\left(\sqrt{z} e_{s}\right) d e_{\lambda} d e_{s},
\end{gathered}
$$

where $\left|\mu_{0}\right|<C\left|q_{\mathrm{mv}}\right|$

\section{THE CAUCHY PROBLEM FOR NAVIER-STOKES' EQUATIONS}

Let us apply the obtained results to estimate the solutions of Cauchy problem for Navier-Stokes' set of equations

$$
\begin{gathered}
q_{t}-v \Delta q+\sum_{k=1}^{3} \uparrow q_{k} q_{x_{k}} \\
=-\nabla p+F_{0}(x, t), \operatorname{div} q=0, \\
\left.q\right|_{t=0}=q_{0}(x)
\end{gathered}
$$

in the domain of $Q_{T}=R^{3} \times(0, T)$. With respect to $q_{0}$, assume

$$
\operatorname{div} q_{0}=0 .
$$

Problem (11), (12), (13) has at least one weak solution (q,p) in the so-called Leray-Hopf class, see [3].

Let us mention the known statements proved in [10].

Theorem 16. Suppose that

$$
q_{0} \in W_{2}^{1}\left(R^{3}\right), \quad f \in L_{2}\left(Q_{T}\right)
$$

then there exists a unique weak solution of problem (11), (12), (13), in $Q_{T_{1}}, T_{1} \in[0, T]$, that satisfies

$$
q_{t}, q_{x x}, \nabla p \in L_{2}\left(Q_{T}\right)
$$

Note that $T_{1}$ depends on $q_{0}, f$.

Lemma 16. If $q_{0} \in W_{2}^{1}\left(R^{3}\right), f \in L_{2}\left(Q_{T}\right)$, then

$$
\begin{aligned}
& \sup _{0 \leq t \leq T}\|q\|_{L_{2}\left(R^{3}\right)}^{2}+\int_{0}^{t} \uparrow\left\|q_{x}\right\|_{L_{2}\left(R^{3}\right)}^{2} d \tau \\
& \leq\left\|q_{0}\right\|_{L_{2}\left(R^{3}\right)}^{2}+\left\|F_{0}\right\|_{L_{2}\left(Q_{T}\right)}
\end{aligned}
$$

Our goal is to prove the global unicity weak solution of (11), (12), (13) irrespective of initial velocity and 
power smallness conditions.

Therefore let us obtain uniform estimates.

Statement 1. Weak solution of problem (11), (12), (13), from Theorem 16 satisfies the following equation

$$
\begin{gathered}
\tilde{q}\left(z\left(e_{k}-e_{\lambda}\right), t\right)=\tilde{q}_{0}\left(z\left(e_{k}-e_{\lambda}\right)\right) \\
+\int_{0}^{t} \nmid e^{-v z^{2}\left|e_{k}-e_{\lambda}\right|(t-\tau)}([(\widetilde{q, \nabla}) q]+\tilde{F}) \\
\times\left(z\left(e_{k}-e_{\lambda}\right), \tau\right) d \tau,
\end{gathered}
$$

where $F=-\nabla p+F_{0}$.

Proof. The proof follows from the definition of Fourier transformation and the formulas for linear differential equations.

Lemma 17. The solution of the problem (11), (12), (13) from Theorem 16, satisfies the following equation

$$
\tilde{p}=\sum_{i, j} \nmid \frac{k_{i} k_{j}}{|k|^{2}} \widetilde{q_{i} q_{j}}+i \sum_{i} \nmid \frac{k_{i}}{|k|^{2}} \widetilde{F_{i}}
$$

and the following estimates

$$
\begin{gathered}
\|p\|_{L_{2}\left(R^{3}\right)} \leq 3\left\|q_{x}\right\|_{L_{2}\left(R^{3}\right)}^{\frac{3}{2}}\|q\|_{L_{2}\left(R^{3}\right)^{\prime}}^{\frac{1}{2}} \\
\left|\frac{\partial \tilde{p}}{\partial k}\right| \leq \frac{\left|\tilde{q}^{2}\right|}{|k|}+\frac{|\tilde{F}|}{|k|^{2}}+\frac{1}{|k|}\left|\frac{\partial \tilde{F}}{\partial k}\right|+3\left|\frac{\partial \tilde{q}^{2}}{\partial|k|}\right| ;
\end{gathered}
$$

Proof. We obtain the equation for $p$ using div and Fourier transformation. The estimates follow from the obtained equation.

This completes the proof of Lemma 17.

Lemma 18. Weak solution of problem (11), (12), (13), from Theorem 16 satisfies the following inequalities

$$
\begin{gathered}
\sup _{0 \leq t \leq T}\left[\left.\int_{R^{3}}|| x\right|^{2}|q(x, t)|^{2} d x\right. \\
\left.+\int_{0}^{t} \uparrow \int_{R^{3}} \uparrow|x|^{2}\left|q_{x}(x, \tau)\right|^{2} d x d \tau\right] \leq \text { const, } \\
\sup _{0 \leq t \leq T}\left[\int_{R^{3}} \uparrow|x|^{4}|q(x, t)|^{2} d x\right. \\
\left.+\int_{0}^{t} \int_{R^{3}} \uparrow|x|^{4}\left|q_{x}(x, \tau)\right|^{2} d x d \tau\right] \leq \text { const, }
\end{gathered}
$$

or

$$
\begin{gathered}
\sup _{0 \leq t \leq T}\left[|| \frac{\partial \tilde{q}}{\partial z}||_{L_{2}\left(R^{3}\right)}\right. \\
+\int_{0}^{t} \uparrow \int_{R^{3}}\left\lceil z^{2}\left|\widetilde{q_{k}}(k, \tau)\right|^{2} d k d \tau\right] \leq \text { const },
\end{gathered}
$$

$$
\begin{gathered}
\sup _{0 \leq t \leq T}\left[|| \frac{\partial^{2} \tilde{q}}{\partial z^{2}}||_{L_{2}\left(R^{3}\right)}\right. \\
\left.+\int_{0}^{t} \uparrow \int_{R^{3}} \uparrow z^{2}\left|\widetilde{q_{k k}}(k, \tau)\right|^{2} d k d \tau\right] \leq \text { const. }
\end{gathered}
$$

Proof. The proof follows from Navier-Stokes' equation, the first priori estimate formulated in Lemma 16 and obtained from Lemma 17.

This completes the proof of Lemma 18.

Lemma 19. Weak solution of problem (11), (12), (13), from Theorem 16, satisfies the following inequalities

$$
\begin{gathered}
\max _{k}|\tilde{q}| \leq \max _{k}\left|\tilde{q}_{0}\right| \\
+\frac{T}{2} \sup _{0 \leq t \leq T}|| q\left|\left\|_{L_{2}\left(R^{3}\right)}^{2}+\int_{0}^{t} \uparrow|| q_{x}\right\|_{L_{2}\left(R^{3}\right)}^{2} d \tau,\right. \\
\max _{k}\left|\frac{\partial \tilde{q}}{\partial z}\right| \leq \max _{k}\left|\frac{\partial \tilde{q}_{0}}{\partial z}\right| \\
+\frac{T}{2} \sup _{0 \leq t \leq T}|| \frac{\partial \tilde{q}}{\partial z}||_{L_{2}\left(R^{3}\right)}+\int_{0}^{t} \uparrow \int_{R^{3}} \nmid z^{2}\left|\widetilde{q_{k}}(k, \tau)\right|^{2} d k d \tau, \\
\max _{k}\left|\frac{\partial^{2} \tilde{q}}{\partial z^{2}}\right| \leq \max _{k}\left|\frac{\partial^{2} \tilde{q}_{0}}{\partial z^{2}}\right| \\
+\frac{T}{2} \sup _{0 \leq t \leq T}|| \frac{\partial^{2} \tilde{q}}{\partial z^{2}}||_{L_{2}\left(R^{3}\right)}+\left.\int_{0} \uparrow \int_{R^{3}}\left|z^{2}\right| \widetilde{q_{k k}}(k, \tau)\right|^{2} d k d \tau .
\end{gathered}
$$

Proof. We obtain these estimates using representation (14), Parseval's equality, Cauchy - Bunyakovskiy inequality (14) by Lemma 18.

This proves Lemma 19.

Lemma 20. Weak solution of problem (11), (12), (13), from Theorem 16 satisfies the following inequalities

$$
\begin{gathered}
\left|\tilde{q}_{\mathrm{mv}}(z, t)\right| \leq z M_{1}, \quad\left|\frac{\partial \tilde{q}_{\mathrm{mv}}(z, t)}{\partial z}\right| \leq z M_{2}, \\
\left|\frac{\partial^{2} \tilde{q}_{\mathrm{mv}}(z, t)}{\partial z^{2}}\right| \leq z M_{3},
\end{gathered}
$$

where $M_{1}, M_{2}, M_{3}$ are limited.

Proof. Let us prove the first estimate. These inequalities

$$
\begin{aligned}
\left|\tilde{q}_{\mathrm{mv}}(z, t)\right| & \leq \frac{z}{2} \int_{0}^{\pi} \uparrow \int_{0}^{2 \pi} \uparrow\left|\tilde{q}\left(z\left(e_{k}-e_{p}\right), t\right)\right| d e_{p} \\
& \leq 2 \pi z \max _{k}|\tilde{q}| \leq z M_{1},
\end{aligned}
$$

where $M_{1}=$ const.

Follows from definition (2) for the average of $q$ and from Lemmas 18, 19.

The rest of estimates are proved similarly. 
This proves Lemma 20.

Lemma 21. Weak solution of problem (11), (12), (13), from Theorem 16 satisfies the following inequalities $C_{i} \leq$ const, $(i=\overline{0,2,4})$, where

$$
\begin{gathered}
C_{0}=\int_{0}^{t} \uparrow\left|\tilde{F}_{1}\right|^{2} d \tau, \quad F_{1}=(q, \nabla) q+F, \\
C_{2}=\int_{0}^{t} \uparrow\left|\frac{\partial \tilde{F}_{1}}{\partial z}\right|^{2} d \tau, \quad C_{4}=\int_{0}^{t} \uparrow\left|\frac{\partial^{2} \tilde{F}_{1}}{\partial z^{2}}\right|^{2} d \tau .
\end{gathered}
$$

The proof follows from the apriori estimate of Lemma 16 and the statement of Lemma 18.

This completes the proof of Lemma 21.

Lemma 22. Suppose that $q \in R, \max _{k}|\tilde{q}|<\infty$, then

$$
\int_{R^{3}} \uparrow \int_{R^{3}} \nmid \frac{q(x) q(y)}{|x-y|^{2}} d x d y \leq C\left(|q|_{L_{2}}+\max _{k}|\tilde{q}|\right)^{2}
$$

Proof. Using Plansherel's theorem, we get the statement of the lemma.

This proves Lemma 22.

Lemma 23. Weak solution of problem (11), (12), (13), from Theorem 16 satisfies the following inequalities

$$
\begin{gathered}
\left|\tilde{q}\left(z\left(e_{k}-e_{\lambda}\right), t\right)\right| \leq\left|\tilde{q}_{0}\left(z\left(e_{k}-e_{\lambda}\right)\right)\right| \\
+\left(\frac{1}{2 v}\right)^{\frac{1}{2}} \frac{C_{0}^{\frac{1}{2}}}{z\left|e_{k}-e_{\lambda}\right|}
\end{gathered}
$$

where

$$
C_{0}=\int_{0}^{t} \rtimes\left|\tilde{F}_{1}\right|^{2} d \tau, F_{1}=(q, \nabla) q+F .
$$

Proof. From Formula (14) we get

where

$$
\begin{gathered}
\left|\tilde{q}\left(z\left(e_{k}-e_{\lambda}\right), t\right)\right| \leq\left|\tilde{q}_{0}\left(z\left(e_{k}-e_{\lambda}\right)\right)\right| \\
+\left|\int_{0}^{t_{\uparrow}}\right| e^{-v z^{2}\left|e_{k}-e_{\lambda}\right|^{2}(t-\tau)} \\
\quad \times \tilde{F}_{1}\left(z\left(e_{k}-e_{\lambda}\right), \tau\right) d \tau \mid
\end{gathered}
$$

$$
F_{1}=(q, \nabla) q+F
$$

Using the denotation

$$
\begin{gathered}
I=\mid \int_{0}^{t}\left\lceil e^{-v z^{2}\left|e_{k}-e_{\lambda}\right|^{2}(t-\tau)}\right. \\
\times \tilde{F}_{1}\left(z\left(e_{k}-e_{\lambda}\right), \tau\right) d \tau \mid,
\end{gathered}
$$

Taking into account Holder's inequality in $I$ we obtain

$$
I \leq\left(\int_{0}^{t} \nmid\left|e^{-v z^{2}\left|e_{k}-e_{\lambda}\right|^{2}(t-\tau)}\right|^{p} d \tau\right)^{\frac{1}{p}}
$$

$$
\times\left(\int_{0}^{t} \uparrow\left|F_{1}\right|^{q} d \tau\right)^{\frac{1}{q}}
$$

where $p, q$ satisfies the equality $\frac{1}{p}+\frac{1}{q}=1$.

Suppose $p=q=2$. Then

$$
I \leq\left(\frac{1}{2 v}\right)^{\frac{1}{2}} \frac{\left(\int_{0}^{t} \uparrow\left|\tilde{F}_{1}\right|^{2} d \tau\right)^{\frac{1}{2}}}{z\left|e_{k}-e_{\lambda}\right|}
$$

Taking into consideration the estimate $I$ in (16), we obtain the statement of the lemma.

This proves Lemma 23.

Now, we have the uniform estimates of Rolnik norms for the solution of problems (11), (12), (13). Our further and basic aim is to get the uniform estimates $\left|\widetilde{q}_{i}\right|_{L_{1}\left(R^{3}\right)}$, a component of velocity components in the Cauchy problem for Navier-Stokes' equations. In order to achieve the aim, we use Theorem 8 it implies to get estimates of spherical average.

Lemma 24. Weak solution of problem (11), (12), (13), from Theorem 16 satisfies the following inequalities

$$
\begin{aligned}
\left|\tilde{q}_{\mathrm{mv}}\right|_{L_{1}\left(R^{3}\right)} \leq \frac{C}{2} & \left(A_{0}^{(1)}+\beta_{1}\left|\tilde{q}_{\mathrm{mv}}\right|_{L_{1}\left(R^{3}\right)}\right) \\
& +|\mu|_{L_{1}\left(R^{3}\right)}
\end{aligned}
$$

the function $\mu$ is defined in Theorem 15 ,

$$
\begin{gathered}
A_{0}^{(1)}=\int_{R^{3}} \nmid z \int_{0}^{\pi} \uparrow \int_{0}^{2 \pi} \uparrow\left|\tilde{q}_{0}\left(z\left(e_{k}-e_{\lambda}\right)\right)\right| \\
\times\left|\tilde{q}_{\mathrm{mv}}\left(z e_{\lambda}, t\right)\right| d e_{\lambda} d k, \beta_{1}=\left(\frac{1}{v}\right)^{\frac{1}{2}} 8 \pi C_{0}^{\frac{1}{2}},
\end{gathered}
$$

and $C_{0}$ is defined in Lemma 23.

Proof. From the statement of Theorem 14, we get the estimate

$$
\begin{gathered}
\left|\tilde{q}_{\mathrm{mv}}\right|_{L_{1}\left(R^{3}\right)} \leq \frac{C}{2} \int_{R^{3}} \nmid z \int_{0}^{\pi} \uparrow \int_{0}^{2 \pi} \uparrow\left|\tilde{q}\left(z\left(e_{k}-e_{\lambda}\right), t\right)\right| \\
\times\left|\tilde{q}_{\mathrm{mv}}\left(z e_{\lambda}, t\right)\right| d e_{\lambda} d k+|\mu|_{L_{1}\left(R^{3}\right)} .
\end{gathered}
$$

(15) in the integral, we obtain

$$
\begin{aligned}
&\left|\tilde{q}_{\mathrm{mv}}\right|_{L_{1}\left(R^{3}\right)} \leq \frac{C}{2}\left(\int_{R^{3}} \uparrow z \int_{0}^{\pi} \uparrow \int_{0}^{2 \pi} \uparrow \mid \tilde{q}_{0}\left(z\left(e_{k}-e_{\lambda}\right) \mid\right.\right. \\
& \times\left|\tilde{q}_{\mathrm{mv}}\left(z e_{\lambda}, t\right)\right| d e_{\lambda} d k \\
&+\left(\frac{1}{v}\right)^{\frac{1}{2}} C_{0}^{\frac{1}{2}} \int_{R^{3}} \uparrow \int_{0}^{\pi} \uparrow \int_{0}^{2 \pi} \uparrow\left|\tilde{q}_{\mathrm{mv}}(k, t)\right| \\
&\left.\quad \times \frac{d e_{\lambda}}{\left|e_{k}-e_{\lambda}\right|} d k\right)+|\mu|_{L_{1}\left(R^{3}\right)} .
\end{aligned}
$$


Let us use the notation

$$
\begin{aligned}
A_{0}^{(1)}= & \int_{R^{3}} \rtimes z \int_{0}^{\pi} \nmid \int_{0}^{2 \pi} \rtimes\left|\tilde{q}_{0}\left(z\left(e_{k}-e_{\lambda}\right)\right)\right| \\
& \times\left|\tilde{q}_{\mathrm{mv}}\left(z e_{\lambda}, t\right)\right| d e_{\lambda} d k
\end{aligned}
$$

then

$$
\begin{gathered}
\left|\tilde{q}_{\mathrm{mv}}\right|_{L_{1}\left(R^{3}\right)} \leq \frac{C}{2}\left(A_{0}^{(1)}+\left(\frac{1}{v}\right)^{\frac{1}{2}} C_{0}^{\frac{1}{2}}\right. \\
\left.\times \int_{R^{3}} \uparrow \int_{0}^{\pi} \uparrow \int_{0}^{2 \pi} \uparrow\left|\tilde{q}_{\mathrm{mv}}(k, t)\right| \frac{d e_{\lambda}}{\left|e_{k}-e_{\lambda}\right|} d k\right)+|\mu|_{L_{1}\left(R^{3}\right)} .
\end{gathered}
$$

Let us use the notation

$$
I_{0}=\int_{0}^{\pi} \uparrow \int_{0}^{2 \pi} \uparrow \frac{d e_{\lambda}}{\left|e_{k}-e_{\lambda}\right|}
$$

and obtain $I_{0}$. Since

$$
\left|e_{k}-e_{\lambda}\right|=\left(\left(e_{k}-e_{\lambda}, e_{k}-e_{\lambda}\right)\right)^{\frac{1}{2}}=(1-\cos \theta)^{\frac{1}{2}}
$$

where $\theta$ is the angle between the unit vectors $e_{k}, e_{\lambda}$, it follows that

$$
I_{0}=4 \pi \int_{0}^{\pi} \uparrow \frac{\sin \theta}{(1-\cos \theta)^{\frac{1}{2}}} d \theta=2^{\frac{7}{2}} \pi .
$$

Using $I_{0}$ in the estimate $\left|\tilde{q}_{\mathrm{mv}}\right|_{L_{1}\left(R^{3}\right)}$, we obtain the statement of the lemma.

This completes the proof of Lemma 24.

Theorem 17. Weak solution of problem (11), (12), (13), from Theorem 16 satisfies the following inequalities

$$
\begin{aligned}
\left|\frac{\tilde{q}_{\mathrm{mv}}}{z}\right|_{L_{1}\left(R^{3}\right)} \leq & \frac{C}{2}\left(A_{0}+\beta_{1}\left|\frac{\tilde{q}_{\mathrm{mv}}}{z}\right|_{L_{1}\left(R^{3}\right)}\right) \\
& +\left|\frac{\mu}{z}\right|_{L_{1}\left(R^{3}\right)^{\prime}}
\end{aligned}
$$

where

$$
\begin{aligned}
A_{0}= & \int_{R^{3}} \uparrow \int_{0}^{\pi} \uparrow \int_{0}^{2 \pi} \uparrow\left|\tilde{q}_{0}\left(z\left(e_{k}-e_{\lambda}\right)\right)\right| \\
& \times\left|\tilde{q}_{\mathrm{mv}}\left(z e_{\lambda}, t\right)\right| d e_{\lambda} d k
\end{aligned}
$$

and $\beta_{1}$ is defined in Lemma 24.

Proof. Proof follows from (16), (17).

Corollary 3. Weak solution of problem (11), (12), (13), from Theorem 16 satisfies the following inequalities

$$
\left|\frac{\tilde{q}_{\mathrm{mv}}}{Z}\right|_{L_{1}\left(R^{3}\right)} \leq\left(\frac{C}{2} A_{0}+\left|\frac{\mu}{Z}\right|_{L_{1}\left(R^{3}\right)}\right) K,
$$

where

$$
K=\frac{v^{\frac{1}{2}}}{v^{\frac{1}{2}}-4 \pi C C_{0}^{\frac{1}{2}}}
$$

Let's consider the influence of the following large scale transformations in Navier-Stokes' equation on $K$

$$
t^{\prime}=t A, \quad v^{\prime}=\frac{v}{A}, \quad v^{\prime}=\frac{v}{A}, \quad F_{0}^{\prime}=\frac{F_{0}}{A^{2}} .
$$

Statement 2. Let

$$
A=\frac{4}{v^{\frac{1}{3}}\left(C C_{0}+1\right)^{\frac{2}{3}}}
$$

then $K \leq \frac{8}{7}$.

Proof. By the definitions $C$ and $C_{0}$, we have

$$
\begin{aligned}
K & =\left(\frac{v}{A}\right)^{\frac{1}{2}}\left(\left(\frac{v}{A}\right)^{\frac{1}{2}}-\frac{4 \pi C C_{0}}{A^{2}}\right)^{-1} \\
& =v^{\frac{1}{2}}\left(v^{\frac{1}{2}}-\frac{4 \pi C C_{0}}{A^{\frac{3}{2}}}\right)^{-1}<\frac{8}{7}
\end{aligned}
$$

This proves Statement 2.

Lemma 25. Weak solution of problem (11), (12), (13), from Theorem 16 satisfies the following inequalities

$$
\begin{gathered}
\left|\frac{\partial \tilde{q}\left(z\left(e_{k}-e_{\lambda}\right), t\right)}{\partial z}\right| \leq\left|\frac{\partial \tilde{q}_{0}\left(z\left(e_{k}-e_{\lambda}\right)\right)}{\partial z}\right| \\
+4 \alpha\left(\frac{1}{v}\right)^{\frac{1}{2}} \frac{C_{0}^{\frac{1}{2}}}{z^{2}\left|e_{k}-e_{\lambda}\right|} \\
+\left(\frac{1}{2 v}\right)^{\frac{1}{2}} \frac{C_{2}^{\frac{1}{2}}}{z\left|e_{k}-e_{\lambda}\right|},
\end{gathered}
$$

where

$$
C_{2}=\int_{0}^{t} \rtimes\left|\frac{\partial \tilde{F}_{1}}{\partial z}\right|^{2} d \tau .
$$

Proof. The underwritten inequalities follows from representation (14)

$$
\begin{gathered}
\left|\frac{\partial \tilde{q}\left(z\left(e_{k}-e_{\lambda}\right), t\right)}{\partial z}\right| \leq\left|\frac{\partial \tilde{q}_{0}\left(z\left(e_{k}-e_{\lambda}\right)\right)}{\partial z}\right| \\
+2 v z\left|e_{k}-e_{\lambda}\right|^{2} \mid \int_{0}^{t} \uparrow(t-\tau) e^{-v z^{2}\left|e_{k}-e_{\lambda}\right|^{2}(t-\tau)} \\
\quad \times \tilde{F}_{1}\left(z\left(e_{k}-e_{\lambda}\right), \tau\right) d \tau \mid \\
+\mid \int_{0}^{t} \nmid e^{-v z^{2}\left|e_{k}-e_{\lambda}\right|^{2}(t-\tau)} \\
\quad \times \frac{\partial \tilde{F}_{1}}{\partial z}\left(z\left(e_{k}-e_{\lambda}\right), \tau\right) d \tau \mid .
\end{gathered}
$$

Let us introduce the following denotation

$$
\begin{aligned}
I_{1}=2 v z \mid e_{k} & -\left.e_{\lambda}\right|^{2} \mid \int_{0}^{t} \nmid(t-\tau) e^{-v z^{2}\left|e_{k}-e_{\lambda}\right|^{2}(t-\tau)} \\
& \times \tilde{F}_{1}\left(z\left(e_{k}-e_{\lambda}\right), \tau\right) d \tau \mid
\end{aligned}
$$




$$
\begin{aligned}
& I_{2}=\mid \int_{0}^{t}\left\lceil e^{-v z^{2}\left|e_{k}-e_{\lambda}\right|^{2}(t-\tau)}\right. \\
& \times \frac{\partial \widetilde{F}_{1}}{\partial z}\left(z\left(e_{k}-e_{\lambda}\right), \tau\right) d \tau \mid
\end{aligned}
$$

then

$$
\begin{aligned}
\left|\frac{\partial \tilde{q}\left(z\left(e_{k}-e_{\lambda}\right), t\right)}{\partial z}\right| \leq\left|\frac{\partial \tilde{q}_{0}\left(z\left(e_{k}-e_{\lambda}\right)\right)}{\partial z}\right| \\
+I_{1}+I_{2} .
\end{aligned}
$$

Estimate $I_{1}$ by means of

$$
\sup _{t}\left|t^{m} e^{-t}\right|<\alpha,
$$

where $m>0$ we obtain

$$
\begin{aligned}
I_{1} & \leq \frac{4 \alpha}{z} \mid \int_{0}^{t} \uparrow e^{-v z^{2}\left|e_{k}-e_{\lambda}\right|^{2} \frac{t-\tau}{2}} \\
& \times \widetilde{F}_{1}\left(z\left(e_{k}-e_{\lambda}\right), \tau\right) d \tau \mid .
\end{aligned}
$$

On applying Holder's inequality, we get

$$
\begin{aligned}
I_{1} \leq \frac{4 \alpha}{Z}\left(\int_{0}^{t} \uparrow\left|e^{-v z^{2}\left|e_{k}-e_{\lambda}\right|^{2} \frac{t-\tau}{2}}\right|^{p} d \tau\right)^{\frac{1}{p}} \\
\times\left(\int_{0}^{t} \uparrow\left|F_{1}\right|^{q} d \tau\right)^{\frac{1}{q}},
\end{aligned}
$$

where $p, q$ satisfy the equality $\frac{1}{p}+\frac{1}{q}=1$.

For $p=q=2$ we have

$$
\begin{gathered}
I_{1} \leq 4 \alpha\left(\frac{1}{v}\right)^{\frac{1}{2}} \frac{C_{0}^{\frac{1}{2}}}{z^{2}\left|e_{k}-e_{\lambda}\right|^{\prime}} \\
I_{2} \leq\left(\frac{1}{2 v}\right)^{\frac{1}{2}} \frac{C_{2}^{\frac{1}{2}}}{z\left|e_{k}-e_{\lambda}\right|^{\prime}} \\
C_{2}=\int_{0}^{t} \uparrow\left|\frac{\partial \tilde{F}_{1}}{\partial z}\right|^{2} d \tau .
\end{gathered}
$$

Inserting $I_{1}, I_{2}$ in to $\left|\frac{\partial \tilde{q}}{\partial z}\right|$, we obtain the statement of the lemma.

This completes the proof of Lemma 25.

Theorem 18. Weak solution of problem (11), (12), (13), from Theorem 16 satisfies the following inequalities

$$
\begin{gathered}
\left|\frac{\partial \tilde{q}_{\mathrm{mv}}}{\partial z}\right|_{L_{1}\left(R^{3}\right)} \leq \frac{C}{2}\left(A_{0}+A_{1}+A_{2}\right. \\
+\beta_{3}\left|\tilde{q}_{\mathrm{mv}}\right|_{L_{1}\left(R^{3}\right)}+\left(\beta_{1}+\beta_{2}\right)\left|\frac{\tilde{q}_{\mathrm{mv}}}{z}\right|_{L_{1}\left(R^{3}\right)} \\
\left.+\beta_{1}\left|\frac{\partial \tilde{q}_{\mathrm{mv}}}{\partial z}\right|_{L_{1}\left(R^{3}\right)}\right)+\left|\frac{\partial \mu}{\partial z}\right|_{L_{1}\left(R^{3}\right)^{\prime}}
\end{gathered}
$$

where

$$
\begin{aligned}
& A_{1}= \int_{R^{3}} \nmid z \int_{0}^{\pi} \uparrow \int_{0}^{2 \pi} \uparrow\left|\frac{\partial \tilde{q}_{0}\left(z\left(e_{k}-e_{\lambda}\right)\right)}{\partial z}\right| \\
& \times\left|\tilde{q}_{\mathrm{mv}}\left(z e_{\lambda}, t\right)\right| d e_{\lambda} d k, \\
& A_{2}= \int_{R^{3}}^{\pi} \int_{0}^{\pi} \uparrow \int_{0}^{2 \pi} \uparrow\left|\tilde{q}_{0}\left(z\left(e_{k}-e_{\lambda}\right)\right)\right| \\
& \times\left|\frac{\partial \tilde{q}_{\mathrm{mv}}\left(z e_{\lambda}, t\right)}{\partial z}\right| d e_{\lambda} d k, \\
& \beta_{2}=\left(\frac{1}{v}\right)^{\frac{1}{2}} 2^{\frac{11}{2}} \pi \alpha C_{0}^{\frac{1}{2}}, \beta_{3}=\left(\frac{1}{v}\right)^{\frac{1}{2}} 8 \pi C_{2}^{\frac{1}{2},}
\end{aligned}
$$

and $C_{2}$ is defined in Lemma 25, $C=$ const.

Proof. From the statement of Theorem 14 we get the following estimate

$$
\begin{aligned}
&\left|\frac{\partial \tilde{q}_{\mathrm{mv}}}{\partial z}\right|_{L_{1}\left(R^{3}\right)} \leq \frac{C}{2}\left(\int_{R^{3}} \uparrow \int_{0}^{\pi} \uparrow \int_{0}^{2 \pi} \uparrow\left|\tilde{q}\left(z\left(e_{k}-e_{\lambda}\right), t\right)\right|\right. \\
& \times\left|\tilde{q}_{\mathrm{mv}}\left(z e_{\lambda}, t\right)\right| d e_{\lambda} d k \\
&+\int_{R^{3}} \nmid z \int_{0}^{\pi} \uparrow \int_{0}^{2 \pi} \uparrow\left|\frac{\partial \tilde{q}\left(z\left(e_{k}-e_{\lambda}\right), t\right)}{\partial z}\right| \\
& \times\left|\tilde{q}_{\mathrm{mv}}\left(z e_{\lambda}, t\right)\right| d e_{\lambda} d k \\
& \times\left\lceil\int_{R^{3}}^{\pi} \int_{0}^{2 \pi} \uparrow\left|\tilde{q}\left(z\left(e_{k}-e_{\lambda}\right), t\right)\right|\right. \\
&\left.\times\left|\frac{\partial \tilde{q}_{\mathrm{mv}}\left(z e_{\lambda}, t\right)}{\partial z}\right| d e_{\lambda} d k\right)+\left|\frac{\partial \mu}{\partial z}\right|_{L_{1}\left(R^{3}\right)} .
\end{aligned}
$$

Let us introduce the following denotation

$$
\begin{aligned}
I_{1}= & \int_{R^{3}} \uparrow \int_{0}^{\pi} \uparrow \int_{0}^{2 \pi} \uparrow\left|\tilde{q}\left(z\left(e_{k}-e_{\lambda}\right), t\right)\right| \\
& \times\left|\tilde{q}_{\mathrm{mv}}\left(z e_{\lambda}, t\right)\right| d e_{\lambda} d k, \\
I_{2}= & \int_{R^{3}} \uparrow z \int_{0}^{\pi} \uparrow \int_{0}^{2 \pi} \uparrow\left|\frac{\partial \tilde{q}\left(z\left(e_{k}-e_{\lambda}\right), t\right)}{\partial z}\right| \\
& \times\left|\tilde{q}_{\mathrm{mv}}\left(z e_{\lambda}, t\right)\right| d e_{\lambda} d k, \\
I_{3}= & \int_{R^{3}} \uparrow z \int_{0}^{\pi} \uparrow \int_{0}^{2 \pi} \uparrow\left|\tilde{q}\left(z\left(e_{k}-e_{\lambda}\right), t\right)\right| \\
& \times\left|\frac{\partial \tilde{q}_{\mathrm{mv}}\left(z e_{\lambda}, t\right)}{\partial z}\right| d e_{\lambda} d k,
\end{aligned}
$$

then

$$
\left|\frac{\partial \tilde{q}_{\mathrm{mv}}}{\partial z}\right|_{L_{1}\left(R^{3}\right)} \leq \frac{C}{2}\left(I_{1}+I_{2}+I_{3}\right)+\left|\frac{\partial \mu}{\partial z}\right|_{L_{1}\left(R^{3}\right)} .
$$

The estimate of $I_{1}$ was obtained in theorem 16, therefore from (15), (18), it follows that 


$$
I_{1} \leq A_{0}+\beta_{1}\left|\frac{\tilde{q}_{\mathrm{mv}}}{z}\right|_{L_{1}\left(R^{3}\right)} .
$$

Inserting inequality (19) into $I_{2}$, we get

$$
\begin{aligned}
& I_{2} \leq \int_{R^{3}} \nmid z \int_{0}^{\pi} \uparrow \int_{0}^{2 \pi} \uparrow\left|\frac{\partial \tilde{q}_{0}\left(z\left(e_{k}-e_{\lambda}\right)\right)}{\partial z}\right| \\
& \times\left|\tilde{q}_{\mathrm{mv}}\left(z e_{\lambda}, t\right)\right| d e_{\lambda} d k \\
&+ 4 \alpha\left(\frac{1}{v}\right)^{\frac{1}{2}} C_{0}^{\frac{1}{2}} I_{0} \int_{R^{3}} \uparrow \frac{\left|\tilde{q}_{\mathrm{mv}}(k, t)\right|}{z} d k \\
&+\left(\frac{1}{2 v}\right)^{\frac{1}{2}} C_{2}^{\frac{1}{2}} I_{0} \int_{R^{3}} \uparrow\left|\tilde{q}_{\mathrm{mv}}(k, t)\right| d k,
\end{aligned}
$$

Let us take into account the estimate of $I_{0}$ obtained in Lemma 25,

$$
I_{0}=\int_{0}^{\pi} \uparrow \int_{0}^{2 \pi} \uparrow \frac{d e_{\lambda}}{\left|e_{k}-e_{\lambda}\right|}=2^{\frac{7}{2}} \pi .
$$

Inserting this value in $I_{2}$, we obtain

$$
\begin{aligned}
& I_{2} \leq \int_{R^{3}} \nmid z \int_{0}^{\pi} \uparrow \int_{0}^{2 \pi} \uparrow\left|\frac{\partial \tilde{q}_{0}\left(z\left(e_{k}-e_{\lambda}\right)\right)}{\partial z}\right| \\
& \quad \times\left|\tilde{q}_{\mathrm{mv}}\left(z e_{\lambda}, t\right)\right| d e_{\lambda} d k \\
& +\left(\frac{1}{v}\right)^{\frac{1}{2}} 2^{\frac{11}{2}} \pi \alpha C_{0}^{\frac{1}{2}} \int_{R^{3}} \nmid \frac{\left|\tilde{q}_{\mathrm{mv}}(k, t)\right|}{z} d k \\
& +\left(\frac{1}{v}\right)^{\frac{1}{2}} 8 \pi C_{2}^{\frac{1}{2}} \int_{R^{3}} \uparrow\left|\tilde{q}_{\mathrm{mv}}(k, t)\right| d k .
\end{aligned}
$$

Let us introduce the following denotation

then

$$
\begin{aligned}
A_{1}= & \int_{R^{3}} \nmid z \int_{0}^{\pi} \uparrow \int_{0}^{2 \pi} \uparrow\left|\frac{\partial \tilde{q}_{0}\left(z\left(e_{k}-e_{\lambda}\right)\right)}{\partial z}\right| \\
& \times\left|\tilde{q}_{\mathrm{mv}}\left(z e_{\lambda}, t\right)\right| d e_{\lambda} d k
\end{aligned}
$$

$$
I_{2} \leq A_{1}+\beta_{2}\left|\frac{\tilde{q}_{\mathrm{mv}}}{z}\right|_{L_{1}\left(R^{3}\right)}+\beta_{3}\left|\tilde{q}_{\mathrm{mv}}\right|_{L_{1}\left(R^{3}\right)},
$$

where

$$
\beta_{2}=\left(\frac{1}{v}\right)^{\frac{1}{2}} 2^{\frac{11}{2}} \pi \alpha C_{0}^{\frac{1}{2}}, \quad \beta_{3}=\left(\frac{1}{v}\right)^{\frac{1}{2}} 8 \pi C_{2}^{\frac{1}{2}} .
$$

Using inequality (16) in $I_{3}$, we get

$$
\begin{aligned}
I_{3} \leq & \int_{R^{3}} \nmid z \int_{0}^{\pi} \uparrow \int_{0}^{2 \pi} \uparrow\left|\tilde{q}_{0}\left(z\left(e_{k}-e_{\lambda}\right)\right)\right| \\
& \times\left|\frac{\partial \tilde{q}_{\mathrm{mv}}\left(z e_{\lambda}, t\right)}{\partial z}\right| d e_{\lambda} d k
\end{aligned}
$$

$$
+\left(\frac{1}{2 v}\right)^{\frac{1}{2}} C_{0}^{\frac{1}{2}} I_{0} \int_{R^{3}} \uparrow\left|\frac{\partial \tilde{q}_{\mathrm{mv}}(k, t)}{\partial z}\right| d k .
$$

Similarly as we estimated $I_{2}$, obtain

$$
I_{3} \leq A_{2}+\beta_{1}\left|\frac{\partial \tilde{q}_{\mathrm{mv}}}{\partial z}\right|_{L_{1}\left(R^{3}\right)^{\prime}}
$$

where

$$
\begin{aligned}
A_{2}= & \int_{R^{3}} \nmid z \int_{0}^{\pi} \uparrow \int_{0}^{2 \pi} \uparrow\left|\tilde{q}_{0}\left(z\left(e_{k}-e_{\lambda}\right)\right)\right| \\
& \times\left|\frac{\partial \tilde{q}_{\mathrm{mv}}\left(z e_{\lambda}, t\right)}{\partial z}\right| d e_{\lambda} d k .
\end{aligned}
$$

Inserting $I_{1}, I_{2}, I_{3}\left|\frac{\partial \tilde{q}_{\mathrm{mv}}}{\partial z}\right|_{L_{1}\left(R^{3}\right)}$, we obtain the statement of the theorem.

This completes the proof of Theorem 18 .

Lemma 26. Weak solution of problem (11), (12), (13), from Theorem 16 satisfies the following inequalities

$$
\begin{gathered}
\left|\frac{\partial^{2} \tilde{q}\left(z\left(e_{k}-e_{\lambda}\right), t\right)}{\partial z^{2}}\right| \leq\left|\frac{\partial^{2} \tilde{q}_{0}\left(z\left(e_{k}-e_{\lambda}\right)\right)}{\partial z^{2}}\right| \\
+\left(\frac{1}{v}\right)^{\frac{1}{2}} \frac{16 \alpha C_{0}^{\frac{1}{2}}}{z^{3}\left|e_{k}-e_{\lambda}\right|}+\left(\frac{1}{v}\right)^{\frac{1}{2}} \frac{8 \alpha C_{2}^{\frac{1}{2}}}{z^{2}\left|e_{k}-e_{\lambda}\right|} \\
+\left(\frac{1}{2 v}\right)^{\frac{1}{2}} \frac{C_{4}^{\frac{1}{2}}}{z\left|e_{k}-e_{\lambda}\right|^{\prime}}
\end{gathered}
$$

where

$$
\sup _{t}\left|t^{m} e^{-t}\right|<\alpha
$$

as $m>0$,

$$
C_{4}=\int_{0}^{t} \nmid\left|\frac{\partial^{2} \tilde{F}_{1}}{\partial z^{2}}\right|^{2} d \tau
$$

Proof. From (14) we have the following inequalities

$$
\begin{aligned}
& \left|\frac{\partial^{2} \tilde{q}\left(z\left(e_{k}-e_{\lambda}\right), t\right)}{\partial z^{2}}\right| \leq\left|\frac{\partial^{2} \tilde{q}_{0}\left(z\left(e_{k}-e_{\lambda}\right)\right)}{\partial z^{2}}\right| \\
& +4 v^{2} z^{2}\left|e_{k}-e_{\lambda}\right|^{4} \mid \int_{0}^{t} \uparrow(t-\tau)^{2} \\
& \times e^{-v z^{2}\left|e_{k}-e_{\lambda}\right|^{2}(t-\tau)} \tilde{F}_{1}\left(z\left(e_{k}-e_{\lambda}\right), \tau\right) d \tau \mid \\
& +4 v z\left|e_{k}-e_{\lambda}\right|^{2} \mid \int_{0}^{t} \uparrow(t-\tau) \\
& \times e^{-v z^{2}\left|e_{k}-e_{\lambda}\right|^{2}(t-\tau)} \frac{\partial \tilde{F}_{1}}{\partial z}\left(z\left(e_{k}-e_{\lambda}\right), \tau\right) d \tau \mid \\
& +\left|\int_{0}^{t} \uparrow e^{-v z^{2}\left|e_{k}-e_{\lambda}\right|^{2}(t-\tau)} \frac{\partial^{2} \tilde{F}_{1}}{\partial z^{2}}\left(z\left(e_{k}-e_{\lambda}\right), \tau\right) d \tau\right| .
\end{aligned}
$$


Let us introduce the following denotation

$$
\begin{gathered}
I_{1}=4 v^{2} z^{2}\left|e_{k}-e_{\lambda}\right|^{4} \mid \int_{0}^{t} \uparrow(t-\tau)^{2} \\
\times e^{-v z^{2}\left|e_{k}-e_{\lambda}\right|^{2}(t-\tau)} \tilde{F}_{1}\left(z\left(e_{k}-e_{\lambda}\right), \tau\right) d \tau \mid, \\
I_{2}=4 v z\left|e_{k}-e_{\lambda}\right|^{2} \mid \int_{0}^{t} \uparrow(t-\tau) \\
\times e^{-v z^{2}\left|e_{k}-e_{\lambda}\right|^{2}(t-\tau)} \frac{\partial \tilde{F}_{1}}{\partial z}\left(z\left(e_{k}-e_{\lambda}\right), \tau\right) d \tau \mid, \\
I_{3}=\mid \int_{0}^{t} \uparrow e^{-v z^{2}\left|e_{k}-e_{\lambda}\right|^{2}(t-\tau)} \\
\times \frac{\partial^{2} \tilde{F}_{1}}{\partial z^{2}}\left(z\left(e_{k}-e_{\lambda}\right), \tau\right) d \tau \mid,
\end{gathered}
$$

then

$$
\begin{gathered}
\left|\frac{\partial^{2} \tilde{q}\left(z\left(e_{k}-e_{\lambda}\right), t\right)}{\partial z^{2}}\right| \leq\left|\frac{\partial^{2} \tilde{q}_{0}\left(z\left(e_{k}-e_{\lambda}\right)\right)}{\partial z^{2}}\right| \\
+I_{1}+I_{2}+I_{3} .
\end{gathered}
$$

Using the estimate

$$
\sup _{t}\left|t^{m} e^{-t}\right|<\alpha
$$

as $m>0$, we estimate $I_{1}, I_{2}$

$$
\begin{aligned}
I_{1} & \leq \frac{16 \alpha}{z^{2}} \mid \int_{0}^{t} \nmid e^{-v z^{2}\left|e_{k}-e_{\lambda}\right|^{2} \frac{t-\tau}{2}} \\
& \times \tilde{F}_{1}\left(z\left(e_{k}-e_{\lambda}\right), \tau\right) d \tau \mid, \\
I_{2} & \leq \frac{8 \alpha}{z} \mid \int_{0}^{t}\left\lceil e^{-v z^{2}\left|e_{k}-e_{\lambda}\right|^{2} \frac{t-\tau}{2}}\right. \\
& \times \frac{\partial \tilde{F}_{1}}{\partial z}\left(z\left(e_{k}-e_{\lambda}\right), \tau\right) d \tau \mid .
\end{aligned}
$$

Using Holder's inequality

$$
\begin{aligned}
& I_{1} \leq \frac{16 \alpha}{Z^{2}}\left(\int_{0}^{t} \uparrow\left|e^{-v z^{2}\left|e_{k}-e_{\lambda}\right|^{2} \frac{t-\tau}{2}}\right|^{p} d \tau\right)^{\frac{1}{p}} \\
& \times\left(\int_{0}^{t} \nmid\left|\tilde{F}_{1}\right|^{q} d \tau\right)^{\frac{1}{q}}, \\
& I_{2} \leq \frac{8 \alpha}{Z}\left(\int_{0}^{t} \uparrow \mid e^{\left.-\left.v z^{2}\left|e_{k}-e_{\lambda}\right|^{2 \frac{t-\tau}{2}}\right|^{p} d \tau\right)^{\frac{1}{p}}}\right. \\
& \times\left(\int_{0}^{t} \uparrow\left|\frac{\partial \tilde{F}_{1}}{\partial z}\right|^{q} d \tau\right)^{\frac{1}{q}},
\end{aligned}
$$

where $p, q$ satisfy the equality $\frac{1}{p}+\frac{1}{q}=1$.
For $p=q=2$ we get

$$
\begin{gathered}
I_{1} \leq 16 \alpha\left(\frac{1}{v}\right)^{\frac{1}{2}} \frac{C_{0}^{\frac{1}{2}}}{z^{3}\left|e_{k}-e_{\lambda}\right|}, \\
I_{2} \leq 8 \alpha\left(\frac{1}{v}\right)^{\frac{1}{2}} \frac{C_{2}^{\frac{1}{2}}}{z^{2}\left|e_{k}-e_{\lambda}\right|} .
\end{gathered}
$$

Taking into account Holder's inequality for $I_{3}$, we get

$$
I_{3} \leq\left(\frac{1}{2 v}\right)^{\frac{1}{2}} \frac{C_{4}^{\frac{1}{2}}}{z\left|e_{k}-e_{\lambda}\right|}, C_{4}=\int_{0}^{t} \uparrow\left|\frac{\partial^{2} \tilde{F}_{1}}{\partial z^{2}}\right|^{2} d \tau .
$$

Inserting $I_{1}, I_{2}, I_{3}$ in $\left|\frac{\partial^{2} \tilde{q}}{\partial z^{2}}\right|$, we get the statement of the lemma.

This completes the proof of Lemma 26.

Theorem 19. Weak solution of problem (11), (12), (13), from Theorem 16 satisfies the following estimate

$$
\begin{gathered}
\left|z \frac{\partial^{2} \tilde{q}_{\mathrm{mv}}}{\partial z^{2}}\right|_{L_{1}\left(R^{3}\right)} \leq \frac{C}{2}\left(2\left(A_{1}+A_{2}+A_{3}\right)\right. \\
+A_{4}+A_{5}+\left(2 \beta_{2}+\beta_{4}\right)\left|\frac{\tilde{q}_{\mathrm{mv}}}{z}\right|_{L_{1}\left(R^{3}\right)} \\
+\left(2 \beta_{3}+\beta_{5}\right)\left|\tilde{q}_{\mathrm{mv}}\right|_{L_{1}\left(R^{3}\right)}+\beta_{6}\left|z \tilde{q}_{\mathrm{mv}}\right|_{L_{1}\left(R^{3}\right)} \\
+2\left(\beta_{1}+\beta_{2}\right)\left|\frac{\partial \tilde{q}_{\mathrm{mv}}}{\partial z}\right|_{L_{1}\left(R^{3}\right)}+2 \beta_{3}\left|z \frac{\partial \tilde{q}_{\mathrm{mv}}}{\partial z}\right|_{L_{1}\left(R^{3}\right)} \\
\left.+\beta_{1}\left|z \frac{\partial^{2} \tilde{q}_{\mathrm{mv}}}{\partial z^{2}}\right|_{L_{1}\left(R^{3}\right)}\right)+\left|z \frac{\partial^{2} \mu}{\partial z^{2}}\right|_{L_{1}\left(R^{3}\right)^{\prime}}(22)
\end{gathered}
$$

where

$$
\begin{aligned}
& A_{3}=\int_{R^{3}} \nmid z^{2} \int_{0}^{\pi} \uparrow \int_{0}^{2 \pi} \uparrow\left|\frac{\partial \tilde{q}_{0}\left(z\left(e_{k}-e_{\lambda}\right)\right)}{\partial z}\right| \\
& \times\left|\frac{\partial \tilde{q}_{\mathrm{mv}}\left(z e_{\lambda}, t\right)}{\partial z}\right| d e_{\lambda} d k, \\
& A_{4}=\int_{R^{3}} \nmid z^{2} \int_{0}^{\pi} \uparrow \int_{0}^{2 \pi} \uparrow\left|\frac{\partial^{2} \tilde{q}_{0}\left(z\left(e_{k}-e_{\lambda}\right)\right)}{\partial z^{2}}\right| \\
& \times\left|\tilde{q}_{\mathrm{mv}}\left(z e_{\lambda}, t\right)\right| d e_{\lambda} d k, \\
& A_{5}=\int_{R^{3}} \nmid z^{2} \int_{0}^{\pi} \uparrow \int_{0}^{2 \pi} \uparrow\left|\tilde{q}_{0}\left(z\left(e_{k}-e_{\lambda}\right)\right)\right| \\
& \times\left|\frac{\partial^{2} \tilde{q}_{\mathrm{mv}}\left(z e_{\lambda}, t\right)}{\partial z^{2}}\right| d e_{\lambda} d k \\
& \beta_{4}=\left(\frac{1}{v}\right)^{\frac{1}{2}} 2^{\frac{15}{2}} \pi \alpha C_{0}^{\frac{1}{2}}, \\
& \beta_{5}=\left(\frac{1}{v}\right)^{\frac{1}{2}} 2^{\frac{13}{2}} \pi \alpha C_{2}^{\frac{1}{2}} \text {, } \\
& \beta_{6}=\left(\frac{1}{v}\right)^{\frac{1}{2}} 8 \pi C_{4}^{\frac{1}{2}}
\end{aligned}
$$


and $C_{4}$ is defined in Lemma 26.

Proof. From the statement of Theorem 14 we have the estimate

$$
\begin{aligned}
& \left|z \frac{\partial^{2} \tilde{q}_{\mathrm{mv}}}{\partial z^{2}}\right|_{L_{1}\left(R^{3}\right)} \leq \frac{C}{2}\left(2 \int_{R^{3}} \nmid z \int_{0}^{\pi} \uparrow \int_{0}^{2 \pi} \uparrow\left|\tilde{q}\left(z\left(e_{k}-e_{\lambda}\right), t\right)\right|\right. \\
& \times\left|\frac{\partial \tilde{q}_{\mathrm{mv}}\left(z e_{\lambda}, t\right)}{\partial z}\right| d e_{\lambda} d k \\
& +2 \int_{R^{3}} \nmid z \int_{0}^{\pi} \uparrow \int_{0}^{2 \pi} \uparrow\left|\frac{\partial \tilde{q}\left(z\left(e_{k}-e_{\lambda}\right), t\right)}{\partial z}\right| \\
& \times\left|\tilde{q}_{\mathrm{mv}}\left(z e_{\lambda}, t\right)\right| d e_{\lambda} d k+ \\
& +2 \int_{R^{3}} \nmid z^{2} \int_{0}^{\pi} \uparrow \int_{0}^{2 \pi} \uparrow\left|\frac{\partial \tilde{q}\left(z\left(e_{k}-e_{\lambda}\right), t\right)}{\partial z}\right| \\
& \times\left|\frac{\partial \tilde{q}_{\mathrm{mv}}\left(z e_{\lambda}, t\right)}{\partial z}\right| d e_{\lambda} d k+ \\
& +\int_{R^{3}} \ngtr z^{2} \int_{0}^{\pi} \uparrow \int_{0}^{2 \pi} \uparrow\left|\frac{\partial^{2} \tilde{q}\left(z\left(e_{k}-e_{\lambda}\right), t\right)}{\partial z^{2}}\right| \\
& \times\left|\tilde{q}_{\mathrm{mv}}\left(z e_{\lambda}, t\right)\right| d e_{\lambda} d k+ \\
& +\int_{R^{3}} \nmid z^{2} \int_{0}^{\pi} \uparrow \int_{0}^{2 \pi} \uparrow\left|\tilde{q}\left(z\left(e_{k}-e_{\lambda}\right), t\right)\right| \\
& \left.\times\left|\frac{\partial^{2} \tilde{q}_{\mathrm{mv}}\left(z e_{\lambda}, t\right)}{\partial z^{2}}\right| d e_{\lambda} d k\right) \\
& +\left|z \frac{\partial^{2} \mu}{\partial z^{2}}\right|_{L_{1}\left(R^{3}\right)}=\frac{C}{2} \sum_{j=1}^{5} \nmid I_{j}+\left|z \frac{\partial^{2} \mu}{\partial z^{2}}\right|_{L_{1}\left(R^{3}\right)} .
\end{aligned}
$$

Let us use the estimates for $I_{1}, I_{2}$

$$
\begin{gathered}
\left.I_{1}=2 \int_{R^{3}}\right\rceil z \int_{0}^{\pi} \uparrow \int_{0}^{2 \pi} \uparrow\left|\tilde{q}\left(z\left(e_{k}-e_{\lambda}\right), t\right)\right| \\
\times\left|\frac{\partial \tilde{q}_{\mathrm{mv}}\left(z e_{\lambda}, t\right)}{\partial z}\right| d e_{\lambda} d k \\
\leq 2\left(A_{1}+\beta_{2}\left|\frac{\tilde{q}_{\mathrm{mv}}}{z}\right|_{L_{1}\left(R^{3}\right)}+\beta_{3}\left|\tilde{q}_{\mathrm{mv}}\right|_{L_{1}\left(R^{3}\right)}\right), \\
I_{2}=2 \int_{R^{3}} \nmid \int_{0}^{\pi} \uparrow \int_{0}^{2 \pi} \uparrow\left|\frac{\partial \tilde{q}\left(z\left(e_{k}-e_{\lambda}\right), t\right)}{\partial z}\right| \\
\times\left|\tilde{q}_{\mathrm{mv}}\left(z e_{\lambda}, t\right)\right| d e_{\lambda} d k \\
\leq 2\left(A_{2}+\beta_{1}\left|\frac{\partial \tilde{q}_{\mathrm{mv}}}{\partial z}\right|_{L_{1}\left(R^{3}\right)}\right) .
\end{gathered}
$$

Let us use inequality (19) to estimate $I_{3}$, then we get

$$
I_{3}=2 \int_{R^{3}} \uparrow z^{2} \int_{0}^{\pi} \uparrow \int_{0}^{2 \pi} \uparrow\left|\frac{\partial \tilde{q}\left(z\left(e_{k}-e_{\lambda}\right), t\right)}{\partial z}\right|
$$

$$
\begin{gathered}
\times\left|\frac{\partial \tilde{q}_{\mathrm{mv}}\left(z e_{\lambda}, t\right)}{\partial z}\right| d e_{\lambda} d k \\
<2\left(\int_{R^{3}}\right\rceil z^{2} \int_{0}^{\pi} \uparrow \int_{0}^{2 \pi} \uparrow\left|\frac{\partial \tilde{q}_{0}\left(z\left(e_{k}-e_{\lambda}\right)\right)}{\partial z}\right| \\
\times\left|\frac{\partial \tilde{q}_{\mathrm{mv}}\left(z e_{\lambda}, t\right)}{\partial z}\right| d e_{\lambda} d k \\
+4 \alpha\left(\frac{1}{v}\right)^{\frac{1}{2}} C_{0}^{\frac{1}{2}} I_{0} \int_{R^{3}}|| \frac{\partial \tilde{q}_{\mathrm{mv}}(k, t)}{\partial z} \mid d k \\
\left.+\left(\frac{1}{2 v}\right)^{\frac{1}{2}} C_{2}^{\frac{1}{2}} I_{0} \int_{R^{3}} \nmid z\left|\frac{\partial \tilde{q}_{\mathrm{mv}}(k, t)}{\partial z}\right| d k\right) .
\end{gathered}
$$

Inserting the value of the integral $I_{0}$, from Lemma 18 , we get

$$
\begin{aligned}
& I_{3}=2\left(\int_{R^{3}} \uparrow z^{2} \int_{0}^{\pi} \uparrow \int_{0}^{2 \pi} \uparrow\left|\frac{\partial \tilde{q}_{0}\left(z\left(e_{k}-e_{\lambda}\right), t\right)}{\partial z}\right|\right. \\
& \times\left|\frac{\partial \tilde{q}_{\mathrm{mv}}\left(z e_{\lambda}, t\right)}{\partial z}\right| d e_{\lambda} d k \\
& +\left(\frac{1}{v}\right)^{\frac{1}{2}} 2^{\frac{11}{2}} \pi \alpha C_{0}^{\frac{1}{2}} \int_{R^{3}} \uparrow\left|\frac{\partial \widetilde{q}_{\mathrm{mv}}(k, t)}{\partial z}\right| d k \\
& \left.+\left(\frac{1}{v}\right)^{\frac{1}{2}} 8 \pi C_{2}^{\frac{1}{2}} \int_{R^{3}} \nmid z\left|\frac{\partial \tilde{q}_{\mathrm{mv}}(k, t)}{\partial z}\right| d k\right) \\
& =2\left(\int_{R^{3}} \uparrow z^{2} \int_{0}^{\pi} \uparrow \int_{0}^{2 \pi} \uparrow\left|\frac{\partial \tilde{q}_{0}\left(z\left(e_{k}-e_{\lambda}\right)\right)}{\partial z}\right|\right. \\
& \times\left|\frac{\partial \tilde{q}_{\mathrm{mv}}\left(z e_{\lambda}, t\right)}{\partial z}\right| d e_{\lambda} d k \\
& \left.+\beta_{2} \int_{R^{3}} \uparrow\left|\frac{\partial \tilde{q}_{\mathrm{mv}}(k, t)}{\partial z}\right| d k+\beta_{3} \int_{R^{3}} \nmid z\left|\frac{\partial \tilde{q}_{\mathrm{mv}}(k, t)}{\partial z}\right| d k\right) .
\end{aligned}
$$

Let us introduce the following denotation

$$
\begin{aligned}
A_{3}= & \int_{R^{3}} \uparrow z^{2} \int_{0}^{\pi} \uparrow \int_{0}^{2 \pi} \uparrow\left|\frac{\partial \tilde{q}_{0}\left(z\left(e_{k}-e_{\lambda}\right)\right)}{\partial z}\right| \\
& \times\left|\frac{\partial \tilde{q}_{\mathrm{mv}}\left(z e_{\lambda}, t\right)}{\partial z}\right| d e_{\lambda} d k,
\end{aligned}
$$

then

$$
\begin{aligned}
I_{3} \leq & 2\left(A_{3}+\beta_{2} \int_{R^{3}} \uparrow\left|\frac{\partial \tilde{q}_{\mathrm{mv}}(k, t)}{\partial z}\right| d k\right. \\
& \left.+\beta_{3} \int_{R^{3}} \uparrow z\left|\frac{\partial \tilde{q}_{\mathrm{mv}}(k, t)}{\partial z}\right| d k\right) .
\end{aligned}
$$

Applying inequality (21) to estimate $I_{4}$, we get 


$$
\begin{aligned}
& I_{4}= \int_{R^{3}} \nmid z^{2} \int_{0}^{\pi} \uparrow \int_{0}^{2 \pi} \uparrow\left|\frac{\partial^{2} \tilde{q}\left(z\left(e_{k}-e_{\lambda}\right), t\right)}{\partial z^{2}}\right| \\
& \times\left|\tilde{q}_{\mathrm{mv}}\left(z e_{\lambda}, t\right)\right| d e_{\lambda} d k \\
& \leq \int_{R^{3}} \nmid z^{2} \int_{0}^{\pi} \uparrow \int_{0}^{2 \pi} \uparrow\left|\frac{\partial^{2} \tilde{q}_{0}\left(z\left(e_{k}-e_{\lambda}\right)\right)}{\partial z^{2}}\right| \\
&+ \times\left(\frac{1}{v}\right)^{\frac{1}{2}} 16 \alpha C_{0}^{\frac{1}{2}} I_{0} \int_{R^{3}} \nmid \frac{1}{z}\left|\tilde{q}_{\mathrm{mv}}(k, t)\right| d k \\
&\left.+\left(\frac{1}{v}\right)^{\frac{1}{2}} 8 \alpha C_{2}^{\frac{1}{2}} I_{0} \int_{R^{3}} \nmid t\right) \mid d e_{\lambda} d k \\
&+\left(\frac{1}{2 v}\right)^{\frac{1}{2}} C_{4}^{\frac{1}{2}} I_{0} \int_{R^{3}}(k, t)|d k| \tilde{q}_{\mathrm{mv}}(k, t) \mid d k .
\end{aligned}
$$

Inserting the value of $I_{0}$, we obtain

$$
\begin{aligned}
& \left.I_{4} \leq \int_{R^{3}}\right\rceil z^{2} \int_{0}^{\pi} \uparrow \int_{0}^{2 \pi} \uparrow\left|\frac{\partial^{2} \tilde{q}_{0}\left(z\left(e_{k}-e_{\lambda}\right)\right)}{\partial z^{2}}\right| \\
& \quad \times\left|\tilde{q}_{\mathrm{mv}}\left(z e_{\lambda}, t\right)\right| d e_{\lambda} d k \\
& +\left(\frac{1}{v}\right)^{\frac{1}{2}} 2^{\frac{15}{2}} \pi \alpha C_{0}^{\frac{1}{2}} \int_{R^{3}} \uparrow \frac{1}{z}\left|\tilde{q}_{\mathrm{mv}}(k, t)\right| d k \\
& \left.+\left(\frac{1}{v}\right)^{\frac{1}{2}} 2^{\frac{13}{2}} \pi \alpha C_{2}^{\frac{1}{2}} \int_{R^{3}}\right\rceil\left|\tilde{q}_{\mathrm{mv}}(k, t)\right| d k \\
& +\left(\frac{1}{2 v}\right)^{\frac{1}{2}} 8 \pi C_{4}^{\frac{1}{2}} \int_{R^{3}} \nmid z\left|\tilde{q}_{\mathrm{mv}}(k, t)\right| d k .
\end{aligned}
$$

Let us introduce the following denotation

$$
\begin{gathered}
\beta_{4}=\left(\frac{1}{v}\right)^{\frac{1}{2}} 2^{\frac{15}{2}} \pi \alpha C_{0}^{\frac{1}{2}}, \beta_{5}=\left(\frac{1}{v}\right)^{\frac{1}{2}} 2^{\frac{13}{2}} \pi \alpha C_{2}^{\frac{1}{2}}, \\
\beta_{6}=\left(\frac{1}{2 v}\right)^{\frac{1}{2}} 8 \pi C_{4}^{\frac{1}{2}},
\end{gathered}
$$

then

$$
\begin{gathered}
I_{4} \leq \int_{R^{3}} \uparrow z^{2} \int_{0}^{\pi} \uparrow \int_{0}^{2 \pi} \uparrow\left|\frac{\partial^{2} \tilde{q}_{0}\left(z\left(e_{k}-e_{\lambda}\right)\right)}{\partial z^{2}}\right| \\
\times\left|\tilde{q}_{\mathrm{mv}}\left(z e_{\lambda}, t\right)\right| d e_{\lambda} d k+\beta_{4} \int_{R^{3}} \uparrow \frac{1}{z}\left|\tilde{q}_{\mathrm{mv}}(k, t)\right| d k \\
+\beta_{5} \int_{R^{3}} \uparrow\left|\tilde{q}_{\mathrm{mv}}(k, t)\right| d k+\beta_{6} \int_{R^{3}} \nmid z\left|\tilde{q}_{\mathrm{mv}}(k, t)\right| d k .
\end{gathered}
$$

Introduce the denotation

$$
\begin{aligned}
A_{4}= & \int_{R^{3}} \uparrow z^{2} \int_{0}^{\pi} \uparrow \int_{0}^{2 \pi} \uparrow\left|\frac{\partial^{2} \tilde{q}_{0}\left(z\left(e_{k}-e_{\lambda}\right)\right)}{\partial z^{2}}\right| \\
& \times\left|\tilde{q}_{\mathrm{mv}}\left(z e_{\lambda}, t\right)\right| d e_{\lambda} d k,
\end{aligned}
$$

then

$$
\begin{gathered}
I_{4} \leq A_{4}+\beta_{4} \int_{R^{3}} \uparrow \frac{1}{Z}\left|\tilde{q}_{\mathrm{mv}}(k, t)\right| d k \\
+\beta_{5} \int_{R^{3}} \uparrow\left|\tilde{q}_{\mathrm{mv}}(k, t)\right| d k+\beta_{6} \int_{R^{3}}\left\lceil z\left|\tilde{q}_{\mathrm{mv}}(k, t)\right| d k .\right.
\end{gathered}
$$

Using inequality (16) to estimate $I_{5}$, we obtain

$$
\begin{aligned}
& I_{5}=\left.\int_{R^{3}}\right\rceil z^{2} \int_{0}^{\pi} \uparrow \int_{0}^{2 \pi} \uparrow\left|\tilde{q}\left(z\left(e_{k}-e_{\lambda}\right), t\right)\right| \\
& \times\left|\frac{\partial^{2} \tilde{q}_{\mathrm{mv}}\left(z e_{\lambda}, t\right)}{\partial z^{2}}\right| d e_{\lambda} d k \\
& \leq\left.\int_{R^{3}}\right\rceil z^{2} \int_{0}^{\pi} \uparrow \int_{0}^{2 \pi} \uparrow\left|\tilde{q}_{0}\left(z\left(e_{k}-e_{\lambda}\right)\right)\right| \\
& \times\left|\frac{\partial^{2} \tilde{q}_{\mathrm{mv}}\left(z e_{\lambda}, t\right)}{\partial z^{2}}\right| d e_{\lambda} d k \\
&+\left(\frac{1}{2 v}\right)^{\frac{1}{2}} C_{0}^{\frac{1}{2}} I_{0} \int_{R^{3}} \nmid z\left|\frac{\partial^{2} \tilde{q}_{\mathrm{mv}}(k, t)}{\partial z^{2}}\right| d k .
\end{aligned}
$$

Inserting the value of the integral $I_{0}$, we obtain

$$
\begin{gathered}
I_{5} \leq \int_{R^{3}} \uparrow z^{2} \int_{0}^{\pi} \uparrow \int_{0}^{2 \pi} \uparrow\left|\tilde{q}_{0}\left(z\left(e_{k}-e_{\lambda}\right)\right)\right| \\
\times\left|\frac{\partial^{2} \tilde{q}_{\mathrm{mv}}\left(z e_{\lambda}, t\right)}{\partial z^{2}}\right| d e_{\lambda} d k+\beta_{1}\left|z \frac{\partial^{2} \tilde{q}_{\mathrm{mv}}}{\partial z^{2}}\right|_{L_{1}\left(R^{3}\right)} .
\end{gathered}
$$

Let us introduce the following denotation

$$
\begin{aligned}
A_{5}= & \int_{R^{3}}\left\lceil z^{2} \int_{0}^{\pi} \uparrow \int_{0}^{2 \pi} \uparrow\left|\tilde{q}_{0}\left(z\left(e_{k}-e_{\lambda}\right)\right)\right|\right. \\
& \times\left|\frac{\partial^{2} \tilde{q}_{\mathrm{mv}}\left(z e_{\lambda}, t\right)}{\partial z^{2}}\right| d e_{\lambda} d k
\end{aligned}
$$

then

$$
I_{5} \leq A_{5}+\beta_{1}\left|z \frac{\partial^{2} \tilde{q}_{\mathrm{mv}}}{\partial z^{2}}\right|_{L_{1}\left(R^{3}\right)} .
$$

Inserting $I_{j},(j=1, \ldots, 5)$ in $\left|z \frac{\partial^{2} \tilde{q}_{\mathrm{mv}}}{\partial z^{2}}\right|_{L_{1}\left(R^{3}\right)}$, we obtain the statement of the theorem.

This completes the proof of Theorem 19.

Lemma 27. Weak solution of problem (11), (12), (13), from Theorem 16 satisfies the following estimate

$$
\begin{aligned}
& \left|\frac{\tilde{q}_{\mathrm{mv}}}{z}\right|_{L_{1}\left(R^{3}\right)} \leq B_{0} K, \\
& \left|\tilde{q}_{\mathrm{mv}}\right|_{L_{1}\left(R^{3}\right)} \leq B_{1} K,
\end{aligned}
$$




$$
\left|z \tilde{q}_{\mathrm{mv}}\right|_{L_{1}\left(R^{3}\right)} \leq B_{2} K
$$

where

$$
\begin{gathered}
K=\frac{v^{\frac{1}{2}}}{v^{\frac{1}{2}}-4 \pi C C_{0}^{\frac{1}{2}}}, B_{0}=\frac{C}{2} A_{0}+\left|\frac{\mu}{Z}\right|_{L_{1}\left(R^{3}\right)}, \\
B_{1}=\frac{C}{2} A_{0}^{(1)}+|\mu|_{L_{1}\left(R^{3}\right)}, \\
B_{2}=\frac{C}{2} A_{0}^{(2)}+|z \mu|_{L_{1}\left(R^{3}\right)}, \\
A_{0}^{(2)}=\int_{R^{3}} \uparrow \int_{0}^{\pi} \uparrow \int_{0}^{2 \pi} \nmid z^{2}\left|\tilde{q}_{0}\left(z\left(e_{k}-e_{\lambda}\right)\right)\right| \\
\times\left|\tilde{q}_{\mathrm{mv}}\left(z e_{\lambda}, t\right)\right| d e_{\lambda} d k .
\end{gathered}
$$

Proof. From inequality (15) and estimate (17), we make the sequence of estimates

$$
\begin{aligned}
\left|z^{n} \tilde{q}_{\mathrm{mv}}\right|_{L_{1}\left(R^{3}\right)} \leq & \frac{C}{2}\left(A_{0}^{(n+1)}+\beta_{1}\left|z^{n} \tilde{q}_{\mathrm{mv}}\right|_{L_{1}\left(R^{3}\right)}\right) \\
& +\left|z^{n} \mu\right|_{L_{1}\left(R^{3}\right)},
\end{aligned}
$$

where

$$
\begin{aligned}
A_{0}^{(n+1)}= & \int_{R^{3}} \uparrow \int_{0}^{\pi} \uparrow \int_{0}^{2 \pi}\left\lceil z^{n+1}\left|\tilde{q}_{0}\left(z\left(e_{k}-e_{\lambda}\right)\right)\right|\right. \\
& \times\left|\tilde{q}_{\mathrm{mv}}\left(z e_{\lambda}, t\right)\right| d e_{\lambda} d k . \\
& \beta_{1}=\left(\frac{1}{v}\right)^{\frac{1}{2}} 8 \pi C_{0}^{\frac{1}{2}},
\end{aligned}
$$

and $n$ is an exponent of $z$. From this recurrence formula, as $n=0, n=-1$, we get estimates (17) and (18) accordingly.

For $n=1$ we have

$$
\begin{aligned}
\left|z \tilde{q}_{\mathrm{mv}}\right|_{L_{1}\left(R^{3}\right)} \leq & \frac{C}{2}\left(A_{0}^{(2)}+\beta_{1}\left|z \tilde{q}_{\mathrm{mv}}\right|_{L_{1}\left(R^{3}\right)}\right) \\
& +|z \mu|_{L_{1}\left(R^{3}\right)} .
\end{aligned}
$$

Considering estimates (17), (18) and the last estimate, we obtain the statement of the lemma.

This proves Lemma 27.

Lemma 28. Weak solution of problem (11), (12), (13), from Theorem 16 satisfies the following estimates

$$
\begin{aligned}
& \left|\frac{\partial \tilde{q}_{\mathrm{mv}}}{\partial z}\right|_{L_{1}\left(R^{3}\right)} \leq D_{0} K^{2}+D_{1} K, \\
& \left|z \frac{\partial \tilde{q}_{\mathrm{mv}}}{\partial z}\right|_{L_{1}\left(R^{3}\right)} \leq D_{2} K^{2}+D_{3} K,
\end{aligned}
$$

where

$$
\begin{aligned}
& D_{0}=\frac{C}{2}\left(\beta_{3}^{(0)} B_{1}+\left(\beta_{1}^{(0)}+\beta_{2}^{(0)}\right) B_{0}\right), \\
& D_{1}=\frac{C}{2}\left(A_{0}+A_{1}+A_{2}\right)+\left|\frac{\partial \mu}{\partial z}\right|_{L_{1}\left(R^{3}\right)}, \\
& D_{2}=\frac{C}{2}\left(\beta_{3}^{(0)} B_{2}+\left(\beta_{1}^{(0)}+\beta_{2}^{(0)}\right) B_{1}\right),
\end{aligned}
$$

$$
\begin{aligned}
& D_{3}=\frac{C}{2}\left(A_{0}^{(1)}+A_{1}^{(1)}+A_{2}^{(1)}\right)+\left|z \frac{\partial \mu}{\partial z}\right|_{L_{1}\left(R^{3}\right)^{\prime}}, \\
& A_{1}^{(1)}= \int_{R^{3}} \nmid z^{2} \int_{0}^{\pi} \nmid \int_{0}^{2 \pi} \nmid\left|\frac{\partial \tilde{q}_{0}\left(z\left(e_{k}-e_{\lambda}\right)\right)}{\partial z}\right| \\
& \times\left|\tilde{q}_{\mathrm{mv}}\left(z e_{\lambda}, t\right)\right| d e_{\lambda} d k, \\
& A_{2}^{(1)}= \int_{R^{3}} \nmid z^{2} \int_{0}^{\pi} \nmid \int_{0}^{2 \pi} \uparrow\left|\tilde{q}_{0}\left(z\left(e_{k}-e_{\lambda}\right)\right)\right| \\
& \times\left|\frac{\partial \tilde{q}_{\mathrm{mv}}\left(z e_{\lambda}, t\right)}{\partial z}\right| d e_{\lambda} d k, \\
& \beta_{1}^{(0)}= \frac{8 \pi C_{0}^{\frac{1}{2}}}{v^{\frac{1}{2}}}, \quad \beta_{2}^{(0)}=\frac{2^{\frac{11}{2}} \pi \alpha C_{0}^{\frac{1}{2}}}{v^{\frac{1}{2}}}, \\
& \beta_{3}^{(0)}=\frac{8 \pi C_{2}^{\frac{1}{2}}}{v^{\frac{1}{2}}},
\end{aligned}
$$

Proof. From inequality (19) and estimate (20), let us make the sequence of estimates

$$
\begin{gathered}
\left|z^{n} \frac{\partial \tilde{q}_{\mathrm{mv}}}{\partial z}\right|_{L_{1}\left(R^{3}\right)} \leq \frac{C}{2}\left(A_{0}^{(n)}+A_{1}^{(n)}+A_{2}^{(n)}\right. \\
+\beta_{3}\left|z^{n} \tilde{q}_{\mathrm{mv}}\right|_{L_{1}\left(R^{3}\right)}+\left(\beta_{1}+\beta_{2}\right)\left|\frac{\tilde{q}_{\mathrm{mv}}}{z^{1-n}}\right|_{L_{1}\left(R^{3}\right)} \\
\left.+\beta_{1}\left|z^{n} \frac{\partial \tilde{q}_{\mathrm{mv}}}{\partial z}\right|_{L_{1}\left(R^{3}\right)}\right)+\left|z^{n} \frac{\partial \mu}{\partial z}\right|_{L_{1}\left(R^{3}\right)^{\prime}}
\end{gathered}
$$

where

$$
\begin{aligned}
A_{0}^{(n)}= & \int_{R^{3}} \uparrow \int_{0}^{\pi} \uparrow \int_{0}^{2 \pi} \nmid z^{n}\left|\tilde{q}_{0}\left(z\left(e_{k}-e_{\lambda}\right)\right)\right| \\
& \times\left|\tilde{q}_{\mathrm{mv}}\left(z e_{\lambda}, t\right)\right| d e_{\lambda} d k, \\
A_{1}^{(n)}= & \int_{R^{3}} \nmid z^{n+1} \int_{0}^{\pi} \uparrow \int_{0}^{2 \pi} \nmid\left|\frac{\partial \tilde{q}_{0}\left(z\left(e_{k}-e_{\lambda}\right)\right)}{\partial z}\right| \\
& \times\left|\tilde{q}_{\mathrm{mv}}\left(z e_{\lambda}, t\right)\right| d e_{\lambda} d k, \\
A_{2}^{(n)}= & \int_{R^{3}} \nmid z^{n+1} \int_{0}^{\pi} \nmid \int_{0}^{2 \pi} \uparrow\left|\tilde{q}_{0}\left(z\left(e_{k}-e_{\lambda}\right)\right)\right| \\
& \times\left|\frac{\partial \tilde{q}_{\mathrm{mv}}\left(z e_{\lambda}, t\right)}{\partial z}\right| d e_{\lambda} d k,
\end{aligned}
$$

and $n$ is an exponent of $z$. From this recurrence formula, we get estimate (17) and (18) for $n=0, n=1$, accordingly. And

$$
\begin{aligned}
& \left|z \frac{\partial \tilde{q}_{\mathrm{mv}}}{\partial z}\right|_{L_{1}\left(R^{3}\right)} \leq \frac{C}{2}\left(A_{0}^{(1)}+A_{1}^{(1)}+A_{2}^{(1)}\right. \\
& +\beta_{3}\left|z \tilde{q}_{\mathrm{mv}}\right|_{L_{1}\left(R^{3}\right)}+\left(\beta_{1}+\beta_{2}\right)\left|\tilde{q}_{\mathrm{mv}}\right|_{L_{1}\left(R^{3}\right)}
\end{aligned}
$$




$$
\left.+\beta_{1}\left|z \frac{\partial \tilde{q}_{\mathrm{mv}}}{\partial z}\right|_{L_{1}\left(R^{3}\right)}\right)+\left|z \frac{\partial \mu}{\partial z}\right|_{L_{1}\left(R^{3}\right)},
$$

Considering estimate (17) and the last estimate, we obtain the statement of the lemma.

This completes the proof of Lemma 28.

Lemma 29. The solution of the problem (11), (12), (13), from Theorem 16, satisfies the following estimate

$$
\begin{gathered}
\left|z \frac{\partial^{2} \tilde{q}_{\mathrm{mv}}}{\partial z^{2}}\right|_{L_{1}\left(R^{3}\right)} \leq P_{0} K^{3} \\
+P_{1} K^{2}+P_{2} K
\end{gathered}
$$

where

$$
\begin{gathered}
P_{0}=C\left(\beta_{3}^{(0)} D_{2}+\left(\beta_{1}^{(0)}+\beta_{2}^{(0)}\right) D_{0}\right), \\
P_{1}=\frac{C}{2}\left(\left(2 \beta_{2}^{(0)}+\beta_{4}^{(0)}\right) B_{0}\right. \\
+\left(2 \beta_{3}^{(0)}+\beta_{5}^{(0)}\right) B_{1}+\beta_{6}^{(0)} B_{2} \\
\left.+2 \beta_{3}^{(0)} D_{3}+2\left(\beta_{1}^{(0)}+\beta_{2}^{(0)}\right) D_{1}\right), \\
P_{2}=\frac{C}{2}\left(2\left(A_{1}+A_{2}+A_{3}\right)\right. \\
\left.+A_{4}+A_{5}\right)+\left|z \frac{\partial^{2} \mu}{\partial z^{2}}\right|_{L_{1}\left(R^{3}\right)}, \\
\beta_{4}^{(0)}=\frac{2^{\frac{15}{2}} \pi \alpha C_{0}^{\frac{1}{2}}}{v^{\frac{1}{2}}}, \quad \beta_{5}^{(0)}=\frac{2^{\frac{13}{2}} \pi \alpha C_{2}^{\frac{1}{2}}}{v^{\frac{1}{2}}}, \\
\beta_{6}^{(0)}=\frac{8 \pi C_{4}^{\frac{1}{2}}}{v^{\frac{1}{2}}} .
\end{gathered}
$$

Proof. From (22), we obtain the following estimate

$$
\begin{gathered}
\left|z \frac{\partial^{2} \tilde{q}_{\mathrm{mv}}}{\partial z^{2}}\right|_{L_{1}\left(R^{3}\right)} \leq \frac{C}{2}\left(2\left(A_{1}+A_{2}+A_{3}\right)\right. \\
+A_{4}+A_{5}+\left(2 \beta_{2}^{(0)}+\beta_{4}(0)\right)\left|\frac{\tilde{q}_{\mathrm{mv}}}{z}\right|_{L_{1}\left(R^{3}\right)} \\
+\left(2 \beta_{3}(0)+\beta_{5}(0)\right)\left|\tilde{q}_{\mathrm{mv}}\right|_{L_{1}\left(R^{3}\right)} \\
+\beta_{6}(0)\left|z \tilde{q}_{\mathrm{mv}}\right|_{L_{1}\left(R^{3}\right)}+ \\
+2\left(\beta_{1}(0)+\beta_{2}(0)\right)\left|\frac{\partial \tilde{q}_{\mathrm{mv}}}{\partial z}\right|_{L_{1}\left(R^{3}\right)} \\
\left.+2 \beta_{3}(0)\left|z \frac{\partial \tilde{q}_{\mathrm{mv}}}{\partial z}\right|_{L_{1}\left(R^{3}\right)}\right)+\left|z \frac{\partial^{2} \mu}{\partial z^{2}}\right|_{L_{1}\left(R^{3}\right)} .
\end{gathered}
$$

Using estimates (23)-(28) in the last inequality, we obtain the statement of the lemma.

This proves Lemma 29.

Theorem 20. The solution of the problem (11), (12), (13), from Theorem 16, satisfies the following estimate

$$
\begin{gathered}
|\tilde{q}|_{L_{1}\left(R^{3}\right)} \leq\left(\gamma_{1} C_{0}+\gamma_{2} C_{0}^{\frac{1}{2}} C_{2}^{\frac{1}{2}}+\gamma_{3} C_{2}\right) K^{3} \\
+\left(\gamma_{4} C_{0}^{\frac{1}{2}}+\gamma_{5} C_{2}^{\frac{1}{2}}+\gamma_{6} C_{4}^{\frac{1}{2}}\right) K^{2} \\
+\left(\gamma_{7} C_{0}^{\frac{1}{2}}+\gamma_{8} C_{2}^{\frac{1}{2}}+\gamma_{9}\right) K
\end{gathered}
$$

where

$$
\begin{gathered}
K=\frac{v^{\frac{1}{2}}}{v^{\frac{1}{2}}-4 \pi C C_{0}^{\frac{1}{2}}}, C_{0}=\int_{0}^{t} \nmid\left|\tilde{F}_{1}\right|^{2} d \tau, \\
F_{1}=(q, \nabla) q+F,
\end{gathered}
$$

$$
C_{2}=\int_{0}^{t} \uparrow\left|\frac{\partial \tilde{F}_{1}}{\partial z}\right|^{2} d \tau, C_{4}=\int_{0}^{t} \uparrow\left|\frac{\partial^{2} \widetilde{F}_{1}}{\partial z^{2}}\right|^{2} d \tau,
$$$$
\gamma_{1}=\frac{C^{2} 2^{3} \pi^{2}}{v}\left(1+2^{\frac{5}{2}}\right) B_{0},
$$$$
\gamma_{2}=\frac{C^{2} 2^{4} \pi^{2}}{v}\left(1+2^{\frac{5}{2}}\right) B_{1}
$$$$
\gamma_{3}=\frac{C^{2} 2^{3} \pi^{2}}{v} B_{2},
$$$$
\gamma_{4}=\frac{C 2^{3} \pi}{v^{\frac{1}{2}}}\left(\left(1+2^{\frac{9}{2}}\right) B_{0}+\left(1+2^{\frac{5}{2}}\right) D_{1}\right) \text {, }
$$$$
\gamma_{5}=\frac{C 2^{3} \pi}{v^{\frac{1}{2}}}\left(\left(1+2^{\frac{3}{2}}\right) B_{1}+D_{3}\right)
$$

$$
\gamma_{6}=\frac{C 2^{3} \pi}{v^{\frac{1}{2}}}
$$

$$
\gamma_{7}=\frac{C 2^{2} \pi}{v^{\frac{1}{2}}}\left(1+2^{\frac{5}{2}}\right) B_{0}, \quad \gamma_{8}=\frac{C 2^{2} \pi}{v^{\frac{1}{2}}} B_{1},
$$

$$
\gamma_{9}=\frac{C}{2}\left(D_{1}+P_{2}\right), B_{0}=\frac{C}{2} A_{0}+\left|\frac{\mu}{z}\right|_{L_{1}\left(R^{3}\right)^{\prime}}
$$$$
B_{1}=\frac{C}{2} A_{0}^{(1)}+|\mu|_{L_{1}\left(R^{3}\right)}, \quad B_{2}=\frac{C}{2} A_{0}^{(2)}+|z \mu|_{L_{1}\left(R^{3}\right)},
$$

$$
\begin{gathered}
D_{1}=\frac{C}{2}\left(A_{0}+A_{1}+A_{2}\right)+\left|\frac{\partial \mu}{\partial z}\right|_{L_{1}\left(R^{3}\right)}, \\
D_{3}=\frac{C}{2}\left(A_{0}^{(1)}+A_{1}^{(1)}+A_{2}^{(1)}\right)+\left|z \frac{\partial \mu}{\partial z}\right|_{L_{1}\left(R^{3}\right)^{\prime}}, \\
P_{2}=\frac{C}{2}\left(2\left(A_{1}+A_{2}+A_{3}\right)\right. \\
\left.+A_{4}+A_{5}\right)+\left|z \frac{\partial^{2} \mu}{\partial z^{2}}\right|_{L_{1}\left(R^{3}\right)}, \\
\frac{C}{2}=\frac{9 \pi}{4(2 \pi)^{3}},
\end{gathered}
$$

the function $\mu$ is defined in Theorem 15.

Proof. From the Theorem 8

$$
|\tilde{q}|_{L_{1}\left(R^{3}\right)} \leq\left|\frac{\tilde{q}_{\mathrm{mv}}}{z}\right|_{L_{1}\left(R^{3}\right)}
$$




$$
+2\left|\frac{\partial \tilde{q}_{\mathrm{mv}}}{\partial z}\right|_{L_{1}\left(R^{3}\right)}+\frac{1}{4}\left|z \frac{\partial^{2} \tilde{q}_{\mathrm{mv}}}{\partial z^{2}}\right|_{L_{1}\left(R^{3}\right)} .
$$

Using estimates (23), (27), (29) in the right side of this inequality, we get

$$
\begin{gathered}
|\tilde{q}|_{L_{1}\left(R^{3}\right)} \leq B_{0} K+2\left(D_{0} K^{2}+D_{1} K\right) \\
+\frac{1}{4}\left(P_{0} K^{3}+P_{1} K^{2}+P_{2} K\right) \\
\leq \frac{1}{4} P_{0} K^{3}+\left(2 D_{0}+P_{1}\right) K^{2}+\left(B_{0}+D_{1}+P_{2}\right) K,
\end{gathered}
$$

where $B_{i}, K$ are defined in Lemma 27, $D_{i}$ is defined in Lemma 28, and $P_{i}$ is defined in Lemma 29. Taking into account these notations and calculating the coefficients at $C_{0}, C_{2}, C_{4}$, we obtain the statement of the theorem.

This proves Theorem 20 .

Lemma 30. The function $\mu$, defined in Theorem 15, satisfies the following estimates

$$
\begin{aligned}
|\mu|_{L_{1}\left(R^{3}\right)} & \leq \text { const, } \quad|z \mu|_{L_{1}\left(R^{3}\right)} \leq \text { const }, \\
\left|\frac{\partial \mu}{\partial z}\right|_{L_{1}\left(R^{3}\right)} & \leq \text { const }, \\
\left|z \frac{\partial \mu}{\partial z}\right|_{L_{1}\left(R^{3}\right)} & \leq \text { const, }\left|z \frac{\partial^{2} \mu}{\partial z^{2}}\right|_{L_{1}\left(R^{3}\right)} \leq \text { const. }
\end{aligned}
$$

Proof. We can get the estimate of cubic members w.r.t. $\tilde{q}$ in $\mu$ if we resume all the methods for estimating square members w.r.t. $\tilde{q}$.

This completes the proof of Lemma 30.

Lemma 31. Weak solution of problem (11), (12), (13), from Theorem 16 satisfies the following estimates

$$
\begin{aligned}
& A_{0} \leq 2 M_{1} \int_{R^{3}} \uparrow\left(\left|\tilde{q}_{0}\left(z e_{k}\right)\right|\right)_{\mathrm{mv}} d k, \\
& A_{0}^{(1)} \leq 2 M_{1} \int_{R^{3}}^{R^{3}} \nmid z\left(\left|\tilde{q}_{0}\left(z e_{k}\right)\right|\right)_{\mathrm{mv}} d k, \\
& A_{0}^{(2)} \leq 2 M_{1} \int_{R^{3}}^{R^{3}} \nmid z^{2}\left(\left|\tilde{q}_{0}\left(z e_{k}\right)\right|\right)_{\mathrm{mv}} d k, \\
& A_{1} \leq 2 M_{1} \int_{R^{3}} \nmid z\left(\left|\frac{\partial \tilde{q}_{0}\left(z e_{k}\right)}{\partial z}\right|\right)_{\mathrm{mv}} d k, \\
& A_{1}^{(1)} \leq 2 M_{1} \int_{R^{3}} \nmid z^{2}\left(\left|\frac{\partial \tilde{q}_{0}\left(z e_{k}\right)}{\partial z}\right|\right)_{\mathrm{mv}} d k, \\
& A_{2} \leq 2 M_{2} \int_{R^{3}} \nmid z\left(\left|\tilde{q}_{0}\left(z e_{k}\right)\right|\right)_{\mathrm{mv}} d k, \\
& \left.A_{2}^{(1)} \leq 2 M_{2} \int_{R^{3}}^{R^{3}}\right\rceil z^{2}\left(\left|\tilde{q}_{0}\left(z e_{k}\right)\right|\right)_{\mathrm{mv}} d k, \\
& A_{3} \leq 2 M_{2} \int_{R^{3}}^{\gamma^{3}}\left\lceil z^{2}\left(\left|\frac{\partial \tilde{q}_{0}\left(z e_{k}\right)}{\partial z}\right|\right)_{\mathrm{mv}} d k\right. \text {, }
\end{aligned}
$$

$$
\begin{gathered}
\left.A_{4} \leq 2 M_{1} \int_{R^{3}}\right\rceil z^{2}\left(\left|\frac{\partial^{2} \tilde{q}_{0}\left(z e_{k}\right)}{\partial z^{2}}\right|\right)_{\mathrm{mv}} d k, \\
A_{5} \leq 2 M_{3} \int_{R^{3}} \nmid z^{2}\left(\left|\tilde{q}_{0}\left(z e_{k}\right)\right|\right)_{\mathrm{mv}} d k .
\end{gathered}
$$

Proof. The proof follows from Lemmas 18, 19, 20. This proves Lemma 31.

Theorem 21. Suppose that

$$
\begin{aligned}
& q_{0} \in W_{2}^{1}\left(R^{3}\right), F_{0} \in L_{2}\left(Q_{T}\right) \text {, } \\
& \tilde{F}_{0} \in L_{1}\left(Q_{T}\right), \frac{\partial \tilde{F}_{0}}{\partial z} \in L_{1}\left(Q_{T}\right), \\
& \frac{\partial^{2} \tilde{F}_{0}}{\partial z^{2}} \in L_{1}\left(Q_{T}\right), \tilde{q}_{0} \in L_{1}\left(R^{3}\right), \\
& I_{j}=\int_{R^{3}} \nmid z^{j-1}\left(\left|\tilde{q}_{0}\left(z e_{k}\right)\right|\right)_{\mathrm{mv}} d k \leq \text { const }, \\
& (j=\overline{1,3}) \text {, } \\
& I_{j}=\int_{R^{3}} \nmid z^{j-3}\left(\left|\frac{\partial \widetilde{q}_{0}\left(z e_{k}\right)}{\partial z}\right|\right)_{\mathrm{mv}} d k \leq \text { const, } \\
& (j=\overline{4,5}) \text {, } \\
& I_{6}=\int_{R^{3}}\left\lceil z^{2}\left(\left|\frac{\partial^{2} \tilde{q}_{0}\left(z e_{k}\right)}{\partial z^{2}}\right|\right)_{\mathrm{mv}} d k \leq\right. \text { const. }
\end{aligned}
$$

Then there exists a unique weak solution of (11), (12), (13), satisfying the following inequalities

$$
\max _{t} \sum_{i=1}^{3} \nmid\left|\widetilde{q}_{i}\right|_{L_{1}\left(R^{3}\right)} \leq \text { const, }
$$

where const depends only on the theorem conditions.

Proof. It is sufficient to get uniform estimates of the maximum $q_{i}$ to prove that the theorem .These obviously follow from the estimate $\left|\widetilde{q}_{i}\right|_{L_{1}\left(R^{3}\right)}$. Uniform estimates allow to extend the rules of the local existence and unicity local to an interval, where they are correct. To estimate the component of velocity, we use statement 2

$$
\begin{gathered}
q_{i}=\frac{q_{i}}{\int_{0}^{T} \uparrow\left\|\mid q_{x}\right\|_{L_{2}\left(R^{3}\right)}^{2} d t+A+1}, \\
A=\frac{4}{v^{\frac{1}{3}}\left(C C_{0}+1\right)^{\frac{2}{3}}} .
\end{gathered}
$$

Using Lemmas 21, 22 for the potential

$$
q_{i}=\frac{q_{i}}{\int_{0}^{T} \times\left\|q_{x}\right\|_{L_{2}\left(R^{3}\right)}^{2} d t+A+1}
$$

We have $N\left(q_{i}\right)<1$, i.e., it is not necessary to take into account normalization numbers when proving the theorem. Now the statetement of the theorem follows from Theorems 20, 17, Lemmas 21, 30, 31 and the conditions of Theorem 21, that give uniform of velocity maxima at a specified interval of time. 
This comletes the proof of Theorem 21.

Note. In the estimate for $\tilde{q}$ the condition $q(0)>1$ is used. This conditioncan be obviated if we use smooth and bounded function $w$ and make all the estimates for $q_{1}=q+w$ such that $q_{1}(0)>1$ is satisfied. Using the function $w$, we also choose the constant A concordant with the constant $\varepsilon$ from Lemma 3 .

Theorem 21 proves the global solvability and unicity of the Cauchy problem for Navier-Stokes' equation.

\section{CONCLUSIONS}

In Introduction we mentioned the authors whose scientific researches we consider appropriate to call the prehistory of this work. The list of these authors may be considerably extended if we enumerate all the predecessors diachronically or by the significance of their contribution into this research. Actually we intended to obtain evident results which were directly and indirectly indicated by these authors in their scientific works. We do not concentrate on the solution to the multi- dimensional problem of quantum scattering theory although it follows from some certain statements proved in this work. In fact, the problem of over-determination in the multi-dimensional inverse problem of quantum scattering theory is obviated since a potential can be defined by amplitude averaging when the amplitude is a function of three variables. In the classic case of the multi- dimensional inverse problem of quantum scattering theory the potential requires restoring with respect to the amplitude that depends on five variables. This obviously leads to the problem of over-determination. Further detalization could have distracted us from the general research line of the work consisting in application of energy and momentum conservation laws in terms of wave functions to the theory of nonlinear equations. This very method we use in solving the problem of the century, the problem of solvability of the Cauchy problem for Navier-Stokes' equations of viscous incompressible fluid. Let us also note the importance of the fact that the laws of momen- tum and energy conservation in terms of wave functions are conservation laws in the micro-world; but in the classic methods of studying nonlinear equations scientists usually use the priori estimates reflecting the conservation laws of macroscopic quantities. We did not focus attention either on obtaining exact estimates dependent on viscosity, lest the calculations be complicated. However, the pilot analysis shows the possibility of applying these estimates to the problem of limiting viscosity transition tending to zero.

\section{REFERENCES}

[1] Durmagambetov, A.A. and Fazylova, L.S. (1997) Some methods of solving nonlinear equations. Herald of the Karaganda University, Publishers KarGU, Karaganda, 1, 6-17.

[2] Durmagambetov, A.A. (1996) Inverse problem of quantum theory of scattering. International Conference on Inverse and III-Posed Problems, Moscow, 57, 27-30.

[3] Leray, J. (1934) Sur le mouvement d'un liquide visquex emp- lissant l'espace. Acta Math, 63, 193-248.

[4] Novikov, R.G. and Henkin $\bar{\partial}$, G.M. (1987) Equation in multidimensional inverse problem of scattering. Success in Mathematics, N3, 93-152.

[5] Faddeev, L.D. (1974) Inverse problem of quantum theory of scattering. Modern Problems of Math-Ematicians, VINITI, 3, 93-180.

[6] Ramm, A.G. (1992) Multidimensional inverse scattering problems. Pitman Monographs and Surveys in Pure and Applied Mathematics, Longman Scientific Technical, Haplow, 51, 379.

[7] Rid, M. and Saymon, B. (1982) Methods of modern mathematic physics. Theory of Scattering, Vol. 3, M.: Mir.

[8] Povzner, A. (1953) About decomposition of functions, into eigenfunctions of operator $-\Delta u+C u$. Mathematics Collection, 32(74), 108-156.

[9] Rid, M. and Saymon, B. (1982) Methods of modern mathematic physics. Analysis of Operators, 4, M.: Mir.

[10] Ladyzhenskaya, O.A. (1970) Mathematic problems of viscous incondensable liquid dynamics. M.: Science. 